\title{
Optimization of a trapezoidal cavity absorber for the Linear Fresnel Reflector
}

\author{
M.A. Moghimi, K.J. Craig* and J.P. Meyer \\ Department of Mechanical and Aeronautical Engineering, University of Pretoria, Pretoria, South \\ Africa $+27-12-420-3515$ \\ * Corresponding author: ken.craig@up.ac.za
}

\begin{abstract}
To increase the efficiency of Concentrated Solar Power (CSP) plants, the use of optimization methods is a current topic of research. This paper focuses on applying an integrated optimization technology to a solar thermal application, more specifically for the optimization of a trapezoidal cavity absorber of an LFR (Linear Fresnel Reflector), also called a Linear Fresnel Collector (LFC), CSP plant. LFR technology has been developed since the 1960s, and while large improvements in efficiencies have been made, there is still room for improvement. Once such area is in the receiver design where the optimal cavity shape, coatings, insulation thickness, absorber pipe selection, layout and spacing always need to be determined for a specific application. This paper uses a commercial tool to find an optimal design for a set of operating conditions. The objective functions that are used to judge the performance of a 2-D cavity are the combined heat loss through convection, conduction and radiation, as well as a wind resistance area. In this paper the effect of absorbed irradiation is introduced in the form of an outer surface of pipe temperature. Seven geometrical parameters are used as design variables. Based on a sample set requiring 79 CFD simulations, a global utopia point is found that minimizes both objectives. The most sensitive parameters were found to be the top insulation thickness and the cavity depth. Based on the results, the Multi-Objective Genetic Algorithm (MOGA) as contained in ANSYS DesignXplorer is shown to be effective in finding candidate optimal designs as well as the utopia point.
\end{abstract}

Keywords: Linear Fresnel Reflector; LFR; CSP; cavity receiver; Response Surface Method optimization; CFD; ANSYS DesignXplorer.

\section{Introduction}

The sun's energy is currently used as power source via the photovoltaic (PV) effect or via thermal systems (solar thermal/Concentrated Solar Power (CSP)). This study focuses on the latter conversion, where the energy of the sun is transferred to a working fluid (also called Heat Transfer Fluid (HTF)) in what is called a receiver, collector or sometimes absorber, depending on the type and implementation. The main types of CSP plants that exist or are in various stages of development and research are: Parabolic Trough Collector (PTC), Parabolic Dish Reflector (PDR), Heliostat Field Collector (HFC), and Linear Fresnel Reflector (LFR).

The advantages of LFR in comparison to PTC can be listed as the following:

1. Easy maintenance, a stationary absorber or receiver (not requiring tracking of the sun's position, hence no requirement for high-pressure joints), separated receiver and reflector system, and a lower height of mirrors.

2. Cheaper due to its inexpensive planar or slightly curved mirrors in comparison to parabolic ones, a simple tracking system for each individual lightweight reflector, its smaller footprint and height (low structural support expenses by near-ground mounted mirrors), lack of high-pressure flexible joints, no heat exchanger necessary (due to direct steam generation in some cases), using conventional glass on the bottom side of the cavity receiver, and minimal manufacturing, operation and maintenance costs.

3. High-ground utilization, location of the primary reflectors could be next to each other, with the added benefit that the shaded area underneath the collector could be considered as either a parking lot or a greenhouse for growth of shadow plants even in arid areas (Haberle et al., (2002) announced that by using the diffuse light and the light reflected on the back of the mirrors (about $300 \mathrm{~W} / \mathrm{m}^{2}$ ), a controlled greenhouse can be implemented in that space). 


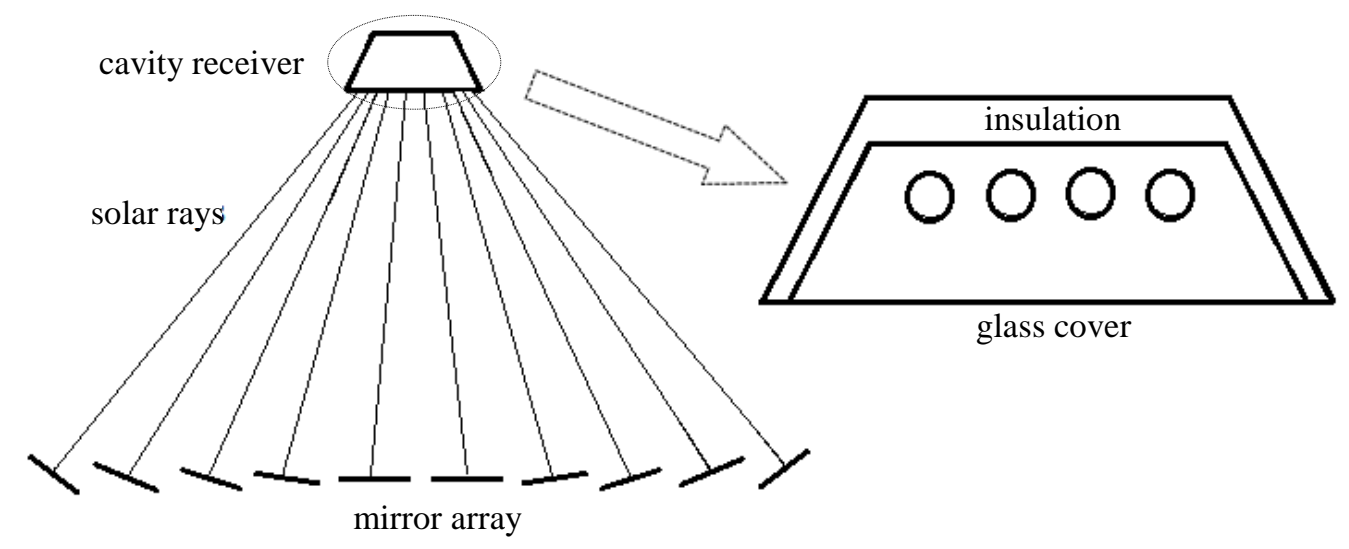

Figure 1: Schematic representation of an LFR solar concentration.

In general, as displayed schematically in Figure 1, an LFR CSP plant is based on an array of linear mirrors that concentrate solar rays on a downward-facing fixed receiver that contains pipe absorbers. These pipes contain a specific working heat transfer fluid (HTF), which is heated by absorbing solar energy. In the current implementation, a glass cover is situated at the aperture to act both as a barrier to heat losses due to external wind conditions, while enhancing the greenhouse effect to trap heat in the cavity. The energy that is absorbed by the HTF is applied to power generation.

Any transfer of energy to an HTF is associated with losses, with thermal losses being the focus of this paper. The concentration of solar rays onto the working fluid causes high local temperatures and re-radiation losses, which are influenced by the geometry of the receiver and the receiver material and optical or surface radiation properties. For a fixed operating condition (e.g., a constant flow rate and inlet temperature), these losses can affect the energy availability for conversion, and hence, the energy output of the thermodynamic cycle is affected. Therefore, the minimization of these losses would directly affect the total efficiency of the CSP plant or the harvesting of freely available solar energy.

Previous researchers conducted studies on various aspects of an LFR cavity receiver to reduce its heat losses. These cavity receivers were considered with and without secondary reflectors, e.g. Abbas et al. (2013) considered a multi-pipe cavity receiver without any secondary reflectors and stated that it was adequate for use in an LFR plant. Trapezoidal cavity receivers without secondary reflectors enhance the performance of an LFR, according to Mills (2004). Other researchers considered different geometrical dimensions. Pye (2008) investigated the performance of receivers ranging from 500 to $1200 \mathrm{~mm}$ for cavity width and 100 - 300 for cavity depth. Lai et al. (2013) also used a trapezoidal geometry, but with a width of $160 \mathrm{~mm}$ and 50-150mm depth. Facão and Oliveira (2011) considered a width of $96 \mathrm{~mm}$ and $25-65 \mathrm{~mm}$ for depth values. Sahoo et al. (2012) referenced their geometry to the pipe diameter, $d$, with the width being $9 d$ and the depth $2.25 \mathrm{~d}-3.75 \mathrm{~d}$. In the above papers, the influence of different parameter variations was reported in individual graphs where the interaction between various variables was not evident. Examples are the influence of cavity depth (Facão and Oliveira, 2011; Lai et al., 2013) or the effect of cavity angle (Pye, 2008) on the thermal loss. These were considered separately instead of as a combination. Another geometrical variable that influences manufacturing is the gap between adjacent pipes and between pipes and adjacent walls (Sahoo et al., 2012). As reported, these gaps influence the fluid flow pattern inside the cavity due to natural convection. The optimal gap size has not been determined by previous researchers.

Besides the geometrical aspects of an LFR, the choice of the insulation around the cavity would influence the thermal performance. The significance of insulation properties (thickness variation, material) in preventing heat losses has rarely been considered. Most of the researchers just considered adiabatic walls instead of modeling insulation (Sahoo et al., 2012), while some researchers (Lin et al., 2013; Sahoo et al., 2013a) considered a fixed insulation thickness. For simple geometries, there is a critical insulation thickness distribution because of the balance between increased thermal resistance with increased thickness and the associated increase in outer contact area for convection and radiation heat transfer losses. For a more complex geometry like the trapezoid considered here, this critical thickness is not uniform and requires optimization. This is especially true because 
of the detrimental effect an increased frontal area would have on wind loads, as considered here.

Based on these studies, it is evident that an optimization study that includes all these variables is justified. The evaluation tools that are used for this study is Computational Fluid Dynamics (CFD) and mathematical optimization. ANSYS Fluent (ANSYS, 2012) is used to determine the conduction, natural convection and thermal re-radiation mechanisms while the receiver geometry, material and surface radiation properties are parameterized and incorporated into a mathematical optimization framework as implemented in ANSYS WorkBench (WB). The framework generates a parameter set (using Design of Experiments (DOE)) based on a mathematical sampling method. Certain output parameters are generated given the parameter set to construct response surfaces. Finally, with the help of an optimization algorithm, optimal results are found on the generated response surfaces. This optimization method is described as the Response Surface Method (RSM).

The aim of this paper is to thermally optimize the cavity of an LFR in a steady-state condition. The cavity is defined as a symmetrical quadrilateral shape containing four HTF pipes. To limit convection losses, a glass window is situated in the cavity aperture. Based on an overall heat balance in a steady-state condition, the heat absorbed in the cavity equals the heat released in the cavity. Like previous researchers, who mostly considered a constant temperature on the HTF pipes' outer walls, the effect of solar irradiation on the pipe outer surfaces is modeled by a constant temperature on the pipes' outer surface. This assumption has been widely used by previous researchers for a single-pipe (Haberle et al., 2002, Heimsath et al., 2014) or a multi-pipe (Pye, 2008; Facão and Oliveira, 2011; Sahoo et al., 2012; Sahoo et al., 2013a; Sahoo et al. 2013b; Lai et al., 2013) LFR receiver. The resulting thermal re-radiation and natural convection (collectively called conjugate heat transfer) is, however, calculated by the CFD model.

The paper first presents the definition of the optimization problem in the context of the cavity layout, and the heat transfer mechanisms considered. This is followed by the optimization formulation and the methodology of the CFD and optimization approach. After a validation section that first evaluates the choice of material property model and then presents a code-to-code comparison validating the assumption of specifying a uniform pipe temperature or flux, the optimization and detailed CFD results are discussed. Finally, conclusions and suggestions for future research are presented.

\section{Definition of cavity layout, heat transfer mechanisms and formulation of optimization problem}

\subsection{Cavity Layout}

A schematic sketch of the proposed trapezoidal cavity for the Liner Fresnel Reflector, that surrounds a parallel four-pipe bundle, is shown in Figure 2. The pipes' OD is held fixed at 50mm to ensure a constant HTF heat transfer area. In addition, the aperture size is held fixed at $1231.3 \mathrm{~mm}$ to satisfy the condition of a given optical system having almost constant intercept factors and a fixed overall output in the optical domain. The other seven geometric parameters shown form part of the design variable set considered in the optimization. The cavity is filled with air. The aperture of the cavity (bottom surface), where irradiation reflected from the LFR mirrors enters, is $3.2 \mathrm{~mm}$ glass (not shown). In order to reduce heat losses from the cavity, the side walls and top wall of the cavity are isolated with a different thickness of insulation. The side and top insulation thicknesses are shown by " $a$ " and " $p$ ", respectively. The pipe pitch and the distance between the pipe center and cavity top wall are shown by " $m$ " and " $d$ ", respectively. In addition, the distance between the center of the outermost pipe and the cavity aperture edge is indicated as " $p$ ". Other geometrical parameters include the cavity depth, "e", and cavity angle and top insulation angle, " $\theta_{1}$ " and " $\theta_{2}$ ", respectively. The overall width of the cavity receiver is denoted as the auxiliary parameter " $2 c$ " in Figure 2. As only a half-symmetrical model is considered in the optimization process, this parameter is reported as " $c$ " in the optimization results. 


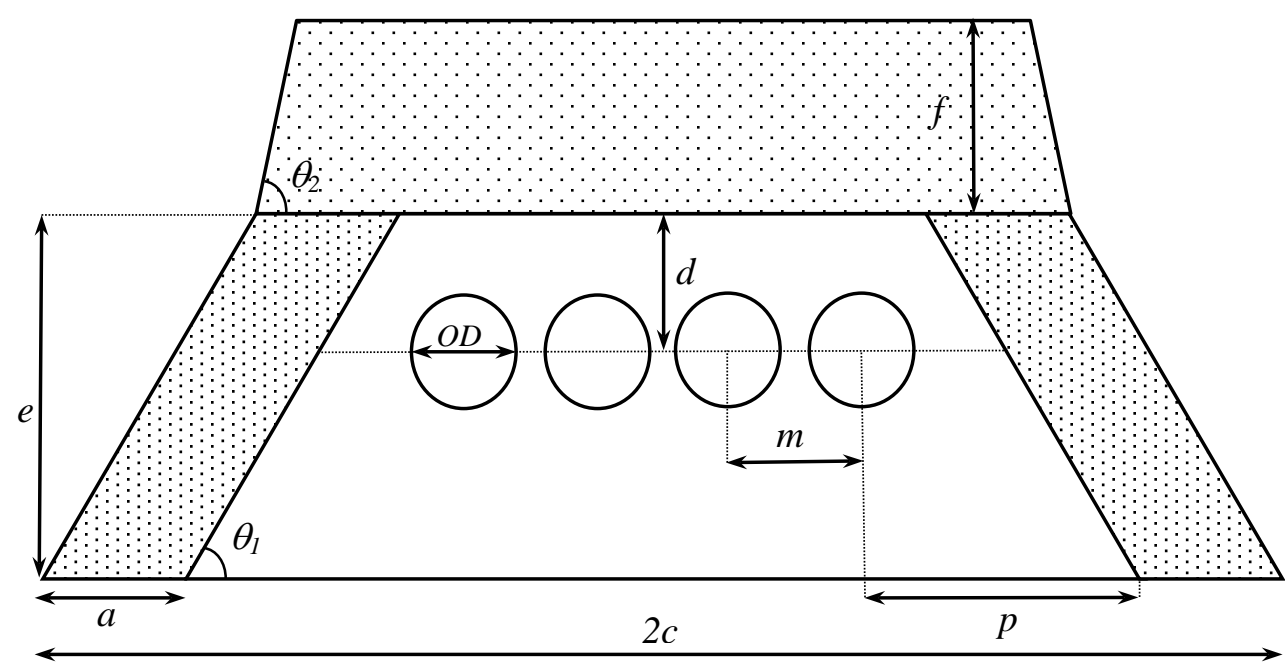

Figure 2: Schematic sketch of proposed cavity receiver.

\subsection{Heat Transfer Mechanisms}

In order to formulate the thermal optimization problem, a thorough understanding of the heat transfer mechanisms present is required. The heat transfer mechanisms in and around the cavity are illustrated in Figure 3. The four absorber pipes are enclosed with a trapezoidal geometry. Typically, high-pressure water, which flows inside the pipes, would absorb the incident solar energy that is irradiated to the cavity by mirrors and passes through the glass cover, which covers the bottom side of the cavity. This energy therefore increases the HTF temperature until it reaches the liquid saturation temperature at the relevant pressure. Beyond this point, the HTF quality increases and a boiling process would occur. In other words, HTF would change from a singlephase flow to a two-phase flow. The HTF could then return to a single phase depending on the conditions, but the only parameter of concern in the current implementation is the average temperature of the pipes' exterior surface. In this paper, a constant pipe outer surface temperature replaces the effect of the solar irradiation absorbed by all the cavity surfaces. This assumption has been used widely by previous researchers as noted above. However, the accuracy of this assumption was not checked in those studies, but it will be in the validation section of this paper.

Inside the cavity domain, all three heat transfer mechanisms exist. Natural convection is driven by temperature differences that cause density differences in the fluid. Because of the gaps between the pipes and the space above and on either side of the pipes, low resistance paths exist for this convection flow to occur. Due to the location of the pipes inside the cavity, one could expect to see such phenomena in the upper part of the cavity domain with conductive heat transfer dominating in the lower half of the cavity, leading to stratification of the temperature in that region. However, radiation remains the dominant heat transfer mechanism in the whole cavity and therefore also the main heat loss contributor. The cavity components (pipes and side and top walls) are considered to be opaque to radiation, i.e., they absorb radiation and reflect it in a way that depends on the incident radiation wavelength (which interacts with the surface roughness height such that reflection is either specular or diffuse). The absorbed energy increases the temperature of each surface, leading to thermal reradiation that is proportional to the emissivity of the surface. It is important to note that the radiative energy which leaves a surface (radiosity) and is combined with the emissive radiation and reflected radiation in a specific direction, then reaches other surfaces where it is defined as the incident radiation. On the other hand, the energy which reaches the cavity bottom surface (covered with glass) is transmitted by conduction and radiation. The glass window has interesting properties. Glass is opaque to high wavelength (infrared) radiation and semitransparent for the rest of the spectrum (see, e.g., glass properties as tested by Loenen and Van der Tempel, 1996). In other words, almost all of the high wavelength incident radiation on glass is absorbed or reflected by this material while for short wavelength incident radiation only a portion of that energy is absorbed and reflected so that the rest passes through this material. This implies that non-grey radiation must be considered in the computational domain. The absorptivity of glass is set at a very high value for the opaque band, while it is low 
for the semi-transparent band. This will be explained in detail in section 3.2.4. To the best authors' knowledge, the investigation of the specular behavior of thermally emitted radiation is one of the novel aspects of this work when modeling solar cavity receivers.

The rest of the domain includes conduction through the insulation, and along its outer edge, an external boundary condition of radiation and convection (due to an external wind) is considered. The insulation, apart from limiting heat transfer across it, is required as its absence would lead to high temperatures on the upper and side walls of the cavity that would emit radiation to the sky temperature, with significant radiation losses.

For simplicity, the heat transfer mechanisms inside the glass, pipes and those over some surfaces inside and outside the cavity have not been shown in Figure 3.

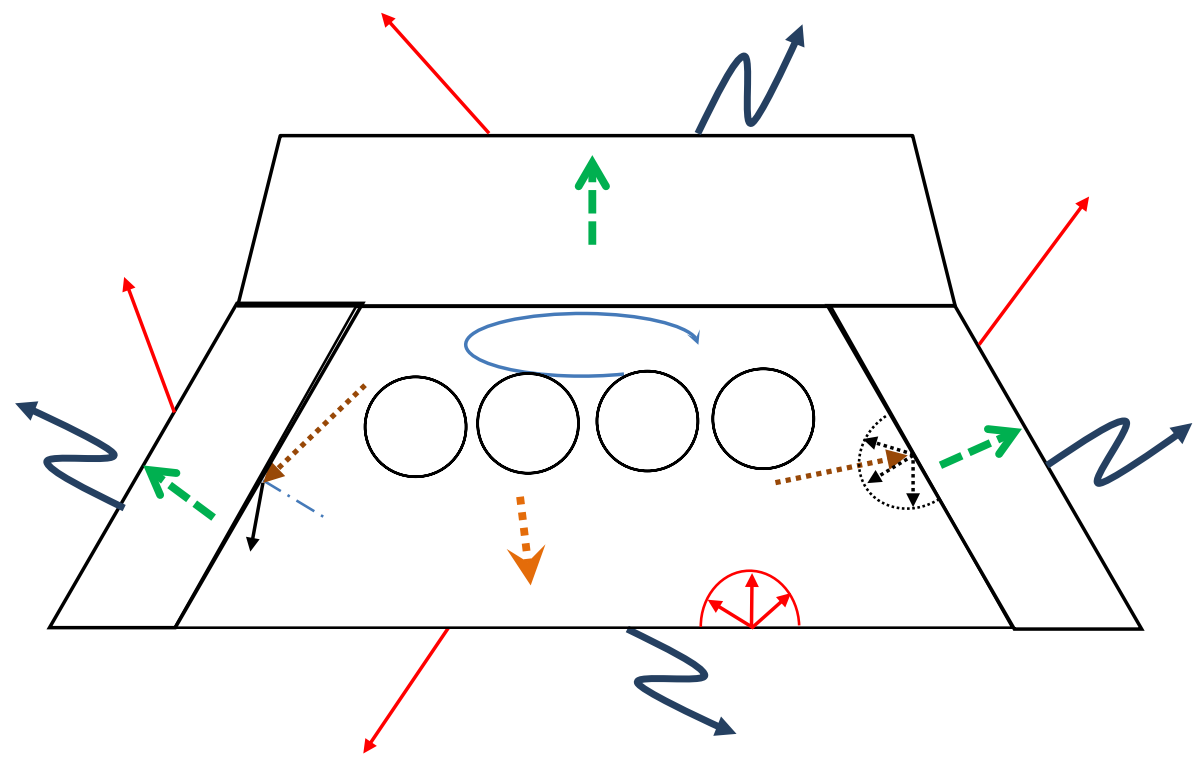

External Forced Convection with ambient Temperature

External radiation to sky Temperature

Conduction inside insulation

Incident radiation

Conductive flow inside cavity

Specular reflection

Convective flow inside cavity

Diffuse emission from a surface inside cavity

Diffuse reflection
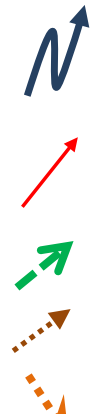

$\because$
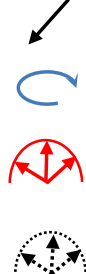

Figure 3: Heat transfer mechanisms for cavity receiver.

\subsection{Formulation of Optimization Problem}

The optimization problem is formulated as follows:

$$
\operatorname{minimize}\left(w_{1} f_{1}(\tilde{x})+w_{2} f_{2}(\tilde{x})\right)=\left(w_{1} \text { Heat loss }(\tilde{x})+w_{2} \operatorname{Area}(\tilde{x})\right)
$$

\section{subject to Bounds on $\tilde{x}$}

with $\tilde{x}=\left(d, e, a, f, m, \theta_{1}, \theta_{2}\right)$

The parameters in the design variable set in Equation 1 are defined in Table 1 . The weighting factors $w_{i}$ are implicitly varied in the multi-objective optimization algorithm defined in section 3.3.1. In essence, the optimization formulation finds the combination of geometrical parameters that define the shape of a trapezoidal LFR cavity that minimize both the heat loss from the cavity as well as its exposed wind area. The geometrical parameters define both the external shape of the cavity (through the definition of the insulation thickness, angular shape and cavity depth) as well as the internal layout (e.g., the relative location of the absorber pipes and pipe distance from top side ) as depicted in Figure 2. The external shape directly influences the wind load (according to Bhaduri and Murphy (1985), the projected area (Figure 4) is directly proportional to the wind load on low-height structures with a height-dependent velocity coefficient also playing a role. For a specific height and velocity as defined by an approaching atmospheric boundary layer, the wind load transferred to the foundation would therefore only vary with projected area). Table 1 also contains derived parameters and the allowable ranges of the independent parameters. 

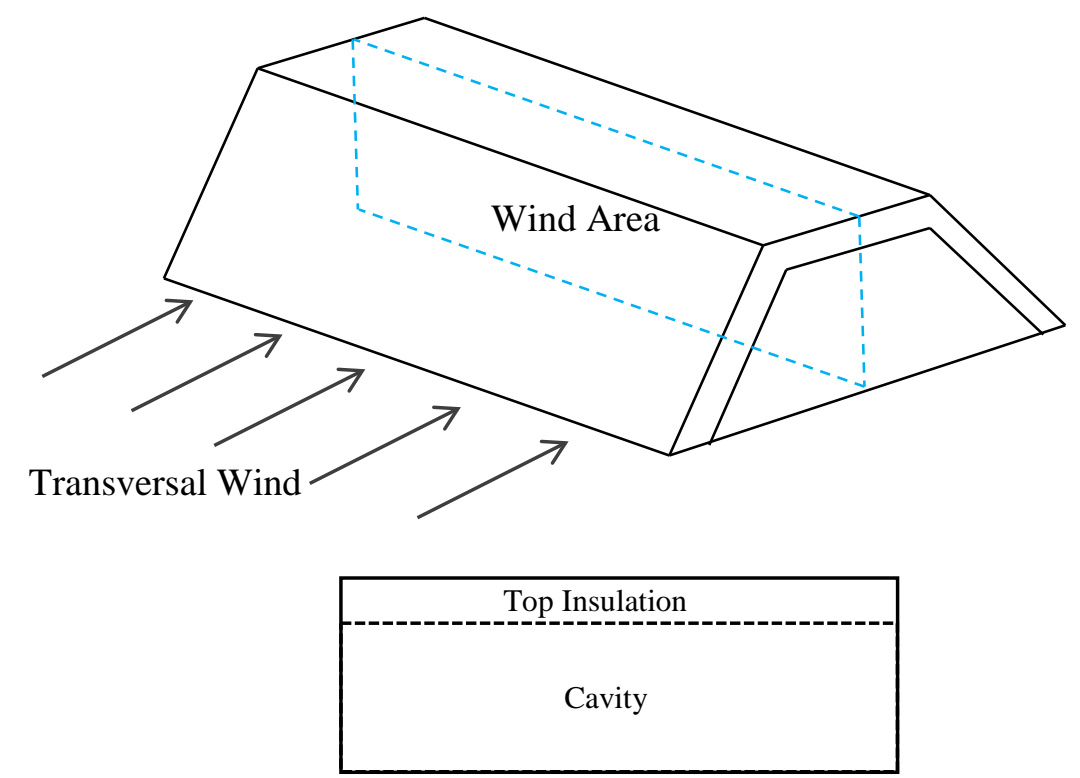

Wind Area

Figure 4: Wind-exposed areas of LFR cavity receiver.

Table 1: Definition of objective and parameter ranges

\begin{tabular}{|c|c|c|}
\hline Independent parameters & Lower bound & Upper bound \\
\hline$d[\mathrm{~mm}]$ & 30 & 105 \\
\hline$e[\mathrm{~mm}]$ & 150 & 300 \\
\hline$a[\mathrm{~mm}]$ & 10 & 100 \\
\hline$f[\mathrm{~mm}]$ & 10 & 95 \\
\hline$m[\mathrm{~mm}]$ & 52 & 75 \\
\hline$\theta_{1}$ & $30^{\circ}$ & $90^{\circ}$ \\
\hline$\theta_{2}$ & $45^{\circ}$ & $90^{\circ}$ \\
\hline Dependent parameters & \multicolumn{2}{|c|}{ Relation } \\
\hline$C$ & \multicolumn{2}{|c|}{$c=0.5($ aperture $+2 a)$} \\
\hline$p$ & \multicolumn{2}{|c|}{$p=.5($ aperture $-3 m)$} \\
\hline$O D[\mathrm{~mm}]$ & \multicolumn{2}{|l|}{50} \\
\hline aperture [mm] & \multicolumn{2}{|c|}{1231.3} \\
\hline Optimization objective & Relation & objective \\
\hline $\begin{array}{l}\text { Heat loss from } \\
\text { boundaries }\end{array}$ & $\begin{array}{l}\text { Summation of heat losses } \\
\text { from outer boundaries }\end{array}$ & Minimization \\
\hline Wind area & $\begin{array}{c}\text { area }=(f+e) * \text { unit length } \\
\text { of cavity receiver }\end{array}$ & Minimization \\
\hline
\end{tabular}

\section{Methodology}

\subsection{Introduction}

In order to minimize the thermal heat losses of an LFR cavity receiver based on CFD simulations, one has to use an optimization tool. There are various optimization tools and software available (e.g., LS-OPT, Isight, Excel, Matlab), which are mostly used as third-party software to interact with commercial CFD codes (e.g. ANSYS Fluent) to optimize a thermo-flow domain and its properties. ANSYS created a platform which allows 
researchers and designers to use its commercial CFD codes in conjunction with Response Surface based optimization tools (DesignXplorer (DX)) within their WorkBench (WB) environment. Parameters for the geometry are defined in the Design Modeler module of WorkBench. The current study uses the ANSYS platform.

In the following, the CFD method and optimization method are explained in more detail in different sections. The first section outlines the mathematical formulation especially as far as radiation modelling is concerned; mesh generation, material and boundary conditions; and the settings used for ANSYS Fluent. The next section explains the optimization method in terms of the optimization algorithms and their settings in DX.

\subsection{CFD Method}

\subsubsection{Conservation Equation}

The LFR cavity was simulated in ANSYS Fluent 15 by considering the following assumptions. The twodimensional model of cavity is in a steady-state condition and the pipe outer surfaces were considered to be at a constant temperature. Moreover, the cavity is filled with air of which the viscosity, heat capacity and thermal conductivity are temperature dependent as defined based on Lienhards' table (Lienhard IV and Lienhard V, 2003). The fluid is considered to be Newtonian and laminar. The no-slip boundary condition for the momentum equation was considered at walls adjacent to the fluid domain. In order to capture natural convection inside the cavity, the density of air was modelled as an incompressible ideal gas where pressure variation is assumed to be insignificant compared to temperature variation. Due to definitions in the heat transfer mechanisms section, almost all energy boundary conditions were considered as coupled. Therefore, by considering the abovementioned assumptions, the transport equations for the fluid flow that are solved on each computational cell or control volume in the mesh are as follows:

$$
\begin{aligned}
& \frac{\partial(\rho u)}{\partial x}+\frac{\partial(\rho v)}{\partial y}=0 \\
& \frac{\partial\left(\rho u^{2}\right)}{\partial x}+\frac{\partial(\rho u v)}{\partial y}=-\frac{\partial(P)}{\partial x}+\frac{\partial}{\partial x}\left(\mu \frac{\partial u}{\partial x}\right)+\frac{\partial}{\partial y}\left(\mu \frac{\partial u}{\partial y}\right) \\
& \frac{\partial(\rho u v)}{\partial x}+\frac{\partial\left(\rho v^{2}\right)}{\partial y}=-\frac{\partial(P)}{\partial y}+\frac{\partial}{\partial x}\left(\mu \frac{\partial v}{\partial x}\right)+\frac{\partial}{\partial y}\left(\mu \frac{\partial v}{\partial y}\right)-g\left(\rho-\rho_{0}\right) \\
& u \frac{\partial\left(\rho c_{p} T\right)}{\partial x}+v \frac{\partial\left(\rho c_{p} T\right)}{\partial y}=\frac{\partial}{\partial x}\left(k \frac{\partial T}{\partial x}\right)+\frac{\partial}{\partial y}\left(k \frac{\partial T}{\partial y}\right)+Q_{\text {radiation }}
\end{aligned}
$$

where the last term in equation 4 is the body-force term, while the last term in equation 5 is the volumetric heat source which is generated by radiation. $x$ and $y$ are the two-dimensional axis directions, $\rho$ the density, $P$ the pressure, $u$ and $v$ the velocity components, $\mu$ the dynamic viscosity, $c_{p}$ the heat capacity at constant pressure, $g$ the gravitational constant, $T$ the temperature and $k$ the thermal conductivity. $\rho_{0}$ is a reference density. ANSYS Fluent defines $\rho$ for the incompressible ideal gas assumption as the following function of temperature:

$$
\rho=\frac{P_{\text {operation }}}{\frac{R}{M_{w}}} \times \frac{1}{T}
$$

where $P_{\text {operation }}$ is the operating pressure, $R$ is the universal gas constant and $M_{w}$ the molecular weight of the gas. When using the incompressible ideal gas assumption for natural convection, one should check after the fact that the pressure variation inside the domain was insignificant in comparison to temperature variation. This check is described in later sections. However, previous researchers analysing LFR cavities mostly used the relatively simple Boussinesq approximation when modelling density in the governing equations of natural convective flow. Pye et al., in different papers (Pye et al. 2003a, 2003b, 2003c), used the Boussinesq approximation for modelling the buoyancy force in fluid flow but he later claimed in his $\mathrm{PhD}$ thesis (Pye, 2008) that "it should be noted that the Boussinesq approximation was used in this cavity modelling, without verification of its accuracy. A calculation using extreme air temperatures of $610 \mathrm{~K}$ and $370 \mathrm{~K}$, and a mean air density of $0.75 \mathrm{~kg} / \mathrm{m} 3(460 \mathrm{~K})$ gives a density variation ratio $\Delta \rho / \rho=0.49$. Further modelling should be performed to quantify the variation in the flow patterns arising from the Boussinesq assumption.” In addition, other researchers (Facão and Oliveira, 2011; Lai et al., 2013; Sahoo et al., 2012, 2013a) used the same approximation without checking its accuracy, 
which might cast doubt on the validity of their results. Guidelines for the applicability of the Boussinesq approximation are given in literature, e.g. when the actual change in density is small (ANSYS, 2012) or when $\beta \Delta \mathrm{T}<<1$ (Bejan, 1993), where $\beta$ is the volumetric thermal expansion coefficient. As CSP cavities often experience large temperature differences, the more accurate incompressible or even compressible ideal gas assumption may be more applicable.

\subsubsection{Radiation Modeling inside domain, radiative surface properties and $S_{N}$ approximation}

\subsubsection{Radiation modeling inside domain}

For solving the transport phenomena equations (equations 2-5), the heat source term, $\left(Q_{\text {radiation}}\right)$, must be determined. As mentioned by Modest (2013), from a physical viewpoint, the net loss of radiative energy from a control volume is equal to emittance of energy from that volume minus the absorbed incident radiation to it. Therefore, the Radiative Transfer Equation (RTE) should be considered in conjunction with the other conservation equations of mass, momentum and energy in order to determine the radiative term, $\left(Q_{\text {radiation }}\right)$. In this study, the air is considered to be a radiatively transparent medium. Two non-gray bands were considered to model both the specular versus diffuse reflection from opaque surfaces due to surface roughness, as well as to model the wavelength-dependent opaqueness of glass. The non-gray radiation behavior was implemented by dividing the radiation spectrum into two wavelength bands with radiation properties remaining fixed in these bands. The bounds of these bands were defined based on typical glass absorption band definitions as 0-4.25 $\mu \mathrm{m}$ and beyond $4.25 \mu \mathrm{m}$ (Dhall et al., 2009). Henceforth, the former one is called the short-wavelength band and the latter the long-wavelength band. The RTE is defined as:

$$
\nabla .\left(I_{\lambda}(\vec{r} . \vec{s}) \vec{s}\right)+\beta I_{\lambda}(\vec{r} . \vec{s})=a_{\lambda} n^{2} I_{b \lambda}+\frac{\sigma_{s}}{4 \pi} \int_{0}^{4 \pi} I_{\lambda}\left(\vec{r} . \vec{s}^{\prime}\right) \phi\left(\vec{s} . \vec{s}^{\prime}\right) d \Omega^{\prime}
$$

In equation 7, the scattering coefficient $\sigma_{s}$, the scattering phase function $\phi$, and the refractive index $n$ are assumed to be independent of wavelength. The terms on the right-hand side describe emission and scattering, while the second term on the left-hand side accounts for the absorption of radiation intensity. $I_{\lambda}$ is the radiation intensity, $a_{\lambda}$ is the absorption coefficient, $\beta$ the combination of the absorption and scattering coefficients, and $\Omega$ ' the solid angle. $I_{b \lambda}$ (the blackbody emission in the wavelength band per unit solid angle) is defined as:

$$
I_{b \lambda}=\left[F\left(0 \rightarrow n \lambda_{2} T\right)-F\left(0 \rightarrow n \lambda_{1} T\right)\right] n^{2} \frac{\sigma T^{4}}{\pi}
$$

where $T$ is the local temperature, $\sigma$ the Stefan-Boltzmann constant, and $F\left(0 \rightarrow n_{2} T\right)$ is the fraction of radiant energy emitted by the blackbody in the wavelength interval from 0 to $\lambda$ as defined by the Planck distribution function or blackbody radiation function (Cengel and Ghajar, 2010). Under the gray assumption, the integral in equation 7 becomes a summation such that the net loss of radiative energy from a control volume is computed as follows:

$$
Q_{\text {radiation }}=-\sum_{k=1}^{n_{\text {last }}} a_{\lambda_{k}}\left(4 \pi I_{b \lambda_{k}}(\vec{r} . \vec{s})-G_{\lambda_{k}}\right) \Delta \lambda_{k}
$$

where $n_{\text {last }}$ is the last defined band number and $G_{\lambda_{k}}$ is the incident radiation function (the total intensity impinging on a point from all sides) which is computed for each band as follows (Modest, 2013):

$$
G_{\lambda}=\int_{4 \pi} I_{\lambda}\left(\vec{s}^{\prime}\right) d \Omega^{\prime}
$$

\subsubsection{Radiative surface properties}

In radiation analysis, four radiative properties (absorptance $\alpha$, emittance $\varepsilon$, reflectance $\rho$ and transmittance $\tau$ ) must be specified for each surface. For local thermal equilibrium of a surface and the adjacent fluid layer, Kirchoff's law reduces the properties to three, because in each wavelength band

$\alpha_{\lambda}=\varepsilon_{\lambda}$.

Based on the definition of the radiative properties of a surface, in each wavelength band, the following relation holds: 
$\alpha_{\lambda}+\rho_{\lambda}+\tau_{\lambda}=1$

The transmission into an opaque surface is zero by definition, implying that for an opaque wall:

$\rho_{\lambda}=1-\varepsilon_{\lambda}$

The reflected energy can be reflected either specularly (in one direction as for a mirror) or diffusely, due to surface roughness. Both types of reflection have the same amount of total energy implying that the diffuse reflection in any direction is less that the total specular amount. In radiation terminology, a rough surface is a surface of which the height of its roughness is much larger than the incident radiation wavelength. So, if the root mean square (RMS) surface roughness is less than incident radiation wavelength, then the surface acts as specular (Bennett and Porteus, 1961), else it acts as diffuse.

In this paper, all opaque surfaces inside the cavity receiver were considered to reflect diffusely in the shortwavelength band and specularly in the long-wavelength band. The glass surface was considered to be highly polished with a negligible surface roughness (RMS 0) (Mellott et al., 2001), and hence specular reflection was prescribed on the glass walls for the whole wavelength domain.

Diffuse and specular reflection is defined in ANSYS Fluent using a diffuse fraction parameter $f_{d}$ for each band.

If $f_{d}$ equals one, it means purely diffuse reflection whereas a zero value implies pure specular reflection for that band.

For, non-gray opaque walls, by combining Kirchoff's law and the radiative surface energy balance, the incident radiative heat flux over a surface would be

$q_{i n, \lambda}=\Delta \lambda \int_{\vec{s} . \vec{n}>0} I_{i n, \lambda} \vec{s} \cdot \vec{n} d \Omega$

where $\vec{n}$ is the surface normal unit vector. The net radiative flux leaving the surface in a band is

$q_{\text {out }, \lambda}=f_{d}\left(1-\varepsilon_{w \lambda}\right) q_{i n, \lambda}+\left(1-f_{d}\right)\left(1-\varepsilon_{w \lambda}\right) q_{i n, \lambda}+\varepsilon_{w \lambda}\left[F\left(0 \rightarrow n \lambda_{2} T\right)-F\left(0 \rightarrow n \lambda_{1} T\right)\right] n^{2} \sigma T_{w}{ }^{4}$

where $\varepsilon_{w \lambda}$ is the wall emissivity in the band. At the wall, the boundary intensity for all outgoing directions in a specific band $\Delta \lambda$ is given by

$I_{0 \lambda}=\frac{q_{\text {out }, \lambda}}{\pi \Delta \lambda}$

It should be considered that for a non-gray diffuse semi-transparent wall, the heat flux on the two sides of a medium is calculated using equations 14 and 15 for each medium, while the incident intensity $I_{\text {in }}$ is calculated from a complicated mathematical equation (omitted for brevity), which is related to the refractive indices for the media.

\subsubsection{2. $\mathrm{S}_{\mathrm{N}}$ approximation}

The numerical procedure which was considered for solving the RTE inside the domain is the Discrete Ordinate Method (DOM) or $\mathrm{S}_{\mathrm{N}}$ approximation as introduced by Modest (2013) and implemented in ANSYS Fluent. The reason for choosing this method for solving the RTE was the capability of DOM to model semi-transparent media. "Ordinate” refers to a direction in hemispherical space. For each ordinate passing in one direction there will be another ordinate in the opposite direction. For that reason, there will be an even number of directions in $\mathrm{S}_{\mathrm{N}}$ approximation.

In a 2-D simulation at any spatial location, each quadrant of angular space is discretized into $N_{\theta} \times N_{\phi}$ of solid angles (control angles) with a fixed vector direction $\vec{s}$ in the global Cartesian system. These control angles are then divided into subdivisions (pixelations). The DOM solves the RTE for a finite number of control angles while the incoming or outgoing radiation to each control angle face is computed by the energy contained in each pixel. In this study, the RTE is uncoupled from the energy equation, implying that at each cell, the RTE and energy equation are solved sequentially with the former implemented through a correction term in the latter. The RTE was solved every 10 iterations of the coupled mass, momentum and energy equation set in order to allow the adjusted radiation heat source to influence the flow in the domain.

\subsubsection{Mesh Generation}


A specific sample geometry for cavity receiver was considered as a starting point for the optimization. The sample geometry dimensions and radiative surface properties were determined based on the selected data of previous researches as summarized in Table 2. This sample geometry was also used in a validation study, to be discussed in Section 4.1. Note that the sample geometry is modeled in its entirety, i.e., symmetry is not used.

\begin{tabular}{|l|l|l|l|l|l|l|l|}
\hline \multicolumn{7}{|c|}{ Table 2: Sample geometry definition } \\
\hline Parameter & Value & Parameter & Value & Parameter & Value & Parameter & Value \\
\hline$\theta_{1}$ & $30^{\circ}$ & $\theta_{2}$ & $60^{\circ}$ & $d$ & $88.34 \mathrm{~mm}$ & $a$ & $40 \mathrm{~mm}$ \\
\hline$m$ & $75 \mathrm{~mm}$ & $c$ & $655.65 \mathrm{~mm}$ & $e$ & $240 \mathrm{~mm}$ & $f$ & $85 \mathrm{~mm}$ \\
\hline
\end{tabular}

The Meshing package of ANSYS WB 15 was used to generate the mesh during the whole optimization process. A triangular mesh with different face sizing elements in each zone was selected for the geometry. The insulation zones, internal cavity domain and glass zone were paved with .012, .007 and .005m triangular element sizes, respectively. In order to provide more freedom for automatic mesh generation during the optimization process, the behavior of these face size elements was set to "Soft". CFD, Fluent and 100 were selected as "Physics Preferences", "Solver Preference” and "Relevance” in the meshing tools. To ensure a smoother mesh with cells concentrated around the pipes and near the cavity walls (due to the high gradients expected in those areas), the "Relevance Center" and "Smoothing" settings were selected as fine, and the "Curvature Normal Angle" and “Growth Rate” were set at $7^{\circ}$ and 1.05 , respectively. Figure 5 depicts the mesh generated for the computational domain. The zoomed-in plot in Figure 5-b illustrates that the mesh concentration around the pipes.

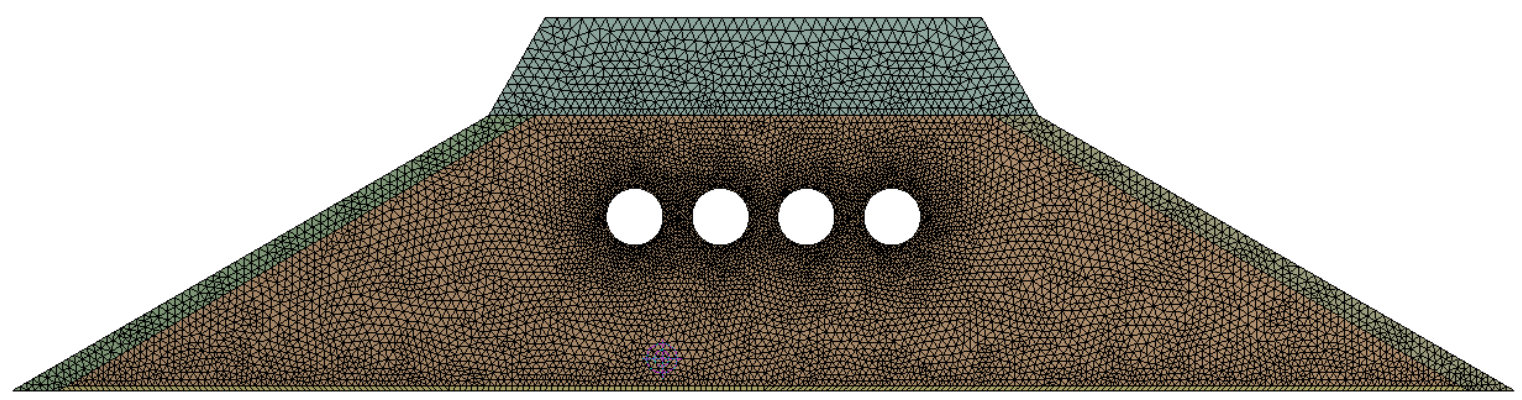

(a)

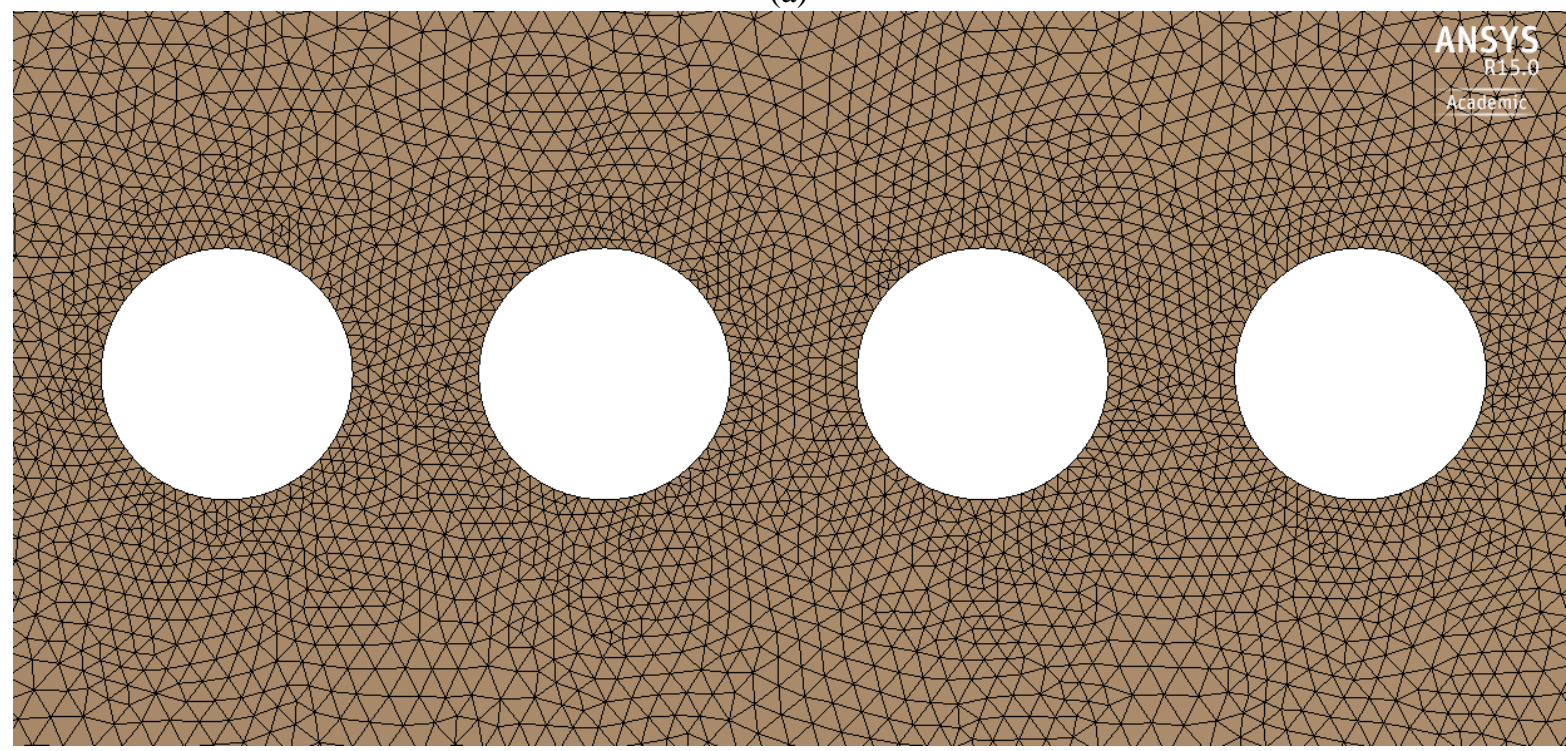

(b)

Figure 5: a) entire zone mesh b) zoomed-in mesh around pipes in cavity. 
A total of 16359 elements were generated in the computational domain. Since poor mesh quality might produce problems in convergence and influence the accuracy of the results, the skewness and aspect ratio of the mesh were checked inside the computational domain. According to Bakker (2013), the skewness should not exceed 0.85 and the local variations in cell size should be minimized and adjacent cells should not have size ratio greater than 1.2 for a high triangular mesh quality. For the proposed problem, the maximum and minimum skewness of elements in the whole domain was 0.5698 and $1.3 \mathrm{e}-6$, respectively, with an average value of $5.7 \mathrm{e}-2$. The maximum and minimum aspect ratio of elements in the whole domain was 1.0008 and 2.5203 , respectively, with average value of 1.1895 . In general, the average element quality in the whole domain was 0.9588 , which provided an excellent mesh quality for the computational domain.

\subsubsection{Material Properties and Boundary Conditions}

The material properties are defined in Table 3. As mentioned before, glass is modeled to be opaque in the highwavelength band. According to Dhall et al. (2009), when using the Beer-Lambert law for a 3.25mm glass thickness, the glass absorption coefficient values should be converted to $29 \%$ and $99 \%$ absorption of wavelengths below and above $4.25 \mu \mathrm{m}$.

\begin{tabular}{|c|c|c|c|c|}
\hline \multicolumn{5}{|c|}{ Table 3: Material properties } \\
\hline Material & $\begin{array}{c}\text { Density } \\
{\left[\mathbf{k g} / \mathbf{m}^{\mathbf{3}}\right]}\end{array}$ & $\begin{array}{c}\text { Specific heat } \\
{[\mathbf{J} / \mathbf{k g}-\mathbf{K}]}\end{array}$ & $\begin{array}{c}\text { Thermal } \\
\text { conductivity } \\
{[\mathbf{W} / \mathbf{m}-\mathbf{K}]}\end{array}$ & Other \\
\hline $\begin{array}{c}\text { Air in cavity } \\
\text { (Lienhard IV and } \\
\text { Lienhard V, 2003) }\end{array}$ & $\begin{array}{c}\text { Incompressible } \\
\text { ideal gas }\end{array}$ & $\begin{array}{c}\text { Piecewise } \\
\text { linear function } \\
\text { of temperature }\end{array}$ & $\begin{array}{c}\text { Piecewise linear } \\
\text { function of } \\
\text { temperature }\end{array}$ & $\begin{array}{c}\text { Viscosity [Pa.s]: } \\
\text { Piecewise linear function } \\
\text { of temperature }\end{array}$ \\
\hline $\begin{array}{c}\text { Pipe-carbon steel } \\
\text { (Incropera et al., } \\
\text { 2006) }\end{array}$ & 7818 & 670 & 54 & - \\
\hline $\begin{array}{c}\text { Semi-transparent } \\
\text { glass (Bansal and } \\
\text { Doremus, 1986) }\end{array}$ & 2650 & 786 & 1.5 & $\begin{array}{c}\text { Refractive index =1.5, } \\
\text { absorption coefficient } \\
{\left[\mathrm{m}^{-1}\right]=106 ; 2300 \text { for }} \\
\text { wavelength below and } \\
\text { above 4.25 } \\
\text { al., 2009) (Dhall et }\end{array}$ \\
\hline $\begin{array}{c}\text { Insulation-glass } \\
\text { wool (TIASA, 2001) }\end{array}$ & 48 & 446 & $\begin{array}{c}\text { Piecewise linear } \\
\text { function of } \\
\text { temperature }\end{array}$ & - \\
\hline
\end{tabular}

For the outer surfaces of the cavity, both convective and radiative thermal boundary conditions are applied. Due to the assumption of external forced convection provided by an approaching wind, a constant convective surface heat transfer coefficient was chosen. The top and side walls were assumed to radiate to sky temperature while the lower cavity surface was assumed to radiate to the LFR mirror temperature, assumed to be $5 \mathrm{~K}$ higher than ambient (Pye, 2008).

The boundary conditions are summarized in Table 4 .

\begin{tabular}{|c|c|c|c|c|}
\hline \multicolumn{5}{|c|}{ Table 4: Boundary condition values } \\
\hline Surface & $\begin{array}{c}\text { Velocity } \\
\text { components } \\
{[\mathbf{m} / \mathbf{s}]}\end{array}$ & $\begin{array}{c}\text { Temperature } \\
{[\mathbf{K}]}\end{array}$ & $\begin{array}{c}\text { Heat transfer } \\
\text { coefficient } \\
{\left[\mathbf{W} / \mathbf{m}^{2}-\mathbf{K}\right]}\end{array}$ & Emissivity \\
\hline Pipe outer side & 0,0 & 500 & - & $\begin{array}{c}0.95 \text { in lower } \\
\text { wavelength band } \\
\text { and 0.1 in higher } \\
\text { wavelength band }\end{array}$ \\
\hline Top, side walls & 0,0 & - & - & 0.05 \\
\hline Glass inner side & 0,0 & - & - & 0.9 (Pye, 2008) \\
\hline
\end{tabular}




\begin{tabular}{|c|c|c|c|c|}
\hline Glass outer side & - & $\begin{array}{c}300 \text { (conv), } \\
305 \text { (rad) }\end{array}$ & 5 & 0.75 \\
\hline Insulation outer & - & 300 (conv), & 5 & 0.75 \\
side & & $\mathrm{T}_{\text {sky }}=0.0522^{*}$ & & \\
& & $300^{1.5}$ (rad) & & \\
& & (Guo et al., & & \\
& & $2014)$ & & \\
\hline
\end{tabular}

\subsubsection{CFD Model Settings}

A 2D model is simulated and parameterized in ANSYS WB 15 (ANSYS, 2013) to predict the total heat loss from the cavity receiver. The laminar, incompressible, convective and radiative fluid flow inside the cavity was solved with the Fluent solver module of ANSYS WB. Natural convection inside the cavity domain was modelled using the incompressible ideal gas assumption. Radiation was modelled using the Discrete Ordinates Method, necessitated by the presence of glass with semi-transparent properties inside the computational domain. Two non-gray bands were considered to model both the specular versus diffuse reflection from opaque surfaces due to surface roughness, as well as to model the wavelength-dependent opaqueness of glass. All emitted (re-)radiation was assumed to be diffuse. Each polar $(\theta)$ and azimuthal $(\phi)$ angle was divided into ten and three divisions as control angles with three subdivisions (pixels) for each angular discretisation, respectively.

The CFD settings strategy included using the SIMPLE algorithm for the first 400 iterations and then switching on the Pressure-Based Coupled Solver with Pseudo-Transient activation for pressure-velocity coupling and solving for 3000 iterations. Then, after a boundary adaptation was implemented using a normal distance of $0.2 \mathrm{~m}$ from all pipe surfaces in the cavity domain, 2500 iterations were solved. Finally, after another adaption in the same region, a final 1500 iterations were run. The spatial discretization of the equations for pressure, momentum, energy and discrete ordinates was chosen as PRESTO, second-order upwind, second-order upwind, and first-order upwind, respectively. This setting strategy led to convergence in all 79 optimization cases by damping all instability in the solutions of these cases due to natural convection, radiation and other factors inherent.

\subsection{Optimization Method}

\subsubsection{Optimization Algorithm}

The following features of ANSYS DesignXplorer (DX) were used for the optimization. The Response Surface Method (RSM) was chosen for the mathematical optimization. The Design of Experiments (DOE) was performed using a Central Composite Design (CCD) of 79 combinations of the 7 independent parameters. The construction of the responses was performed using Gaussian Progress regression, or Kriging. The determination of the optimum location on these surfaces was performed using the Multi-Objective Genetic Algorithm (MOGA), which is based on the Fast and Elitist evolutionary algorithm: NSGA-II (Non-dominated Sorted Genetic Algorithm-II). This method results in a Pareto optimal set given competing objectives. This set represents the family of optimal designs on the combined objective response surfaces through the variation of the relative weighting of the competing objectives. The salient features of Kriging and MOGA are summarized in the following.

\subsubsection{Kriging}

Kriging is named after D.G. Krige who utilized empirical methods for determination of a true ore grade distribution from sample ore grade distribution (LS-OPT, 2003). The basic postulate of its formulation is a combination of a polynomial model plus departures:

$y(x)=f(x)+Z(x)$

where $y(x)$ is the unknown function of interest, $f(x)$ is a known polynomial function, and $Z(x)$ is the stochastic component with mean zero, variance $\sigma^{2}$, and non-zero covariance as listed in equation 18. $f(x)$ approximates the global design space while localized deviations are generated by $Z(x)$. 
$\operatorname{Cov}\left[Z\left(x^{i}\right), Z\left(x^{j}\right)\right\rfloor=\sigma^{2} R\left(\left\lfloor r\left(x^{i}, x^{j}\right)\right]\right)$

with an $M$ number of sampling points, $R$ is the correlation matrix ( $M^{*} M$ symmetric positive definite with unit diagonal matrix) and $r\left(x^{i}, x^{j}\right)$ is the spatial correlation of the function between any two arbitrary sample points $\left(x^{i}, x^{j}\right)$.

The commonly used correlation functions used are Exponential (equation 19-a) and Gaussian (equation 19-b) forms:

$$
\begin{aligned}
& r=\prod_{k=1}^{N} e^{-\vartheta_{k}\left|x_{k}^{i}-x_{k}^{j}\right|} \\
& r=\prod_{k=1}^{N} e^{-\vartheta_{k}\left(x_{k}^{i}-x_{k}^{j}\right)^{2}}
\end{aligned}
$$

where $N$ is number of design variables, and $x_{k}^{i}, x_{k}^{j}$ are the $k^{\text {th }}$ components of two arbitrary sample points. There are thus $M$ number of unknown $\vartheta$ parameters which must be set to fit the model. The correlation which ANSYS DX uses is the Gaussian function (equation 19-b). Using a single correlation parameter does not always provide good results, therefore, ANSYS DX provides an option which lets users choose between a single correlation parameter (one correlation parameter for all design variables), or multiple correlation variables (one correlation

parameter for each design variable), which means that only one unknown $\vartheta$ parameter exists. The choice is made by selecting the Variable or Constant option for the Kernel Variation Type in DX.

$Z(x)$ can be written as follows (ANSYS, 2013b)

$Z(x)=\sum_{i=1}^{N} \lambda_{i} r\left(x^{i}, x\right)$

\subsubsection{MOGA}

Consider $\min _{x \in X} \vec{f}(x)$, where $\vec{f}$ is a multi-objective function. All feasible solutions are defined by:

$X:=\left\{x \in \mathfrak{R}^{\prime \prime}: g(x) \geq 0, h(x)=0, x_{i} \leq x \leq x_{u}\right\}$

where $x$ is the vector of design parameters (or variables) and $x^{\prime} \in X$ such that for all $m$ objective functions, $x^{*}$ is optimal, then

$$
f_{i}\left(x^{*}\right) \leq f_{i}(x) \forall x \in X \quad \text { for } i=1,2, \ldots \ldots, m
$$

This indicates that $x^{*}$ is the utopian solution. Unfortunately, such a solution rarely exists, since the probability of the event that all $f_{i}(x)$ reach their minimum values at a common point $\left(x^{*}\right)$ in $X$ would be rare. The concept of a Pareto optimum was defined by V. Pareto in 1986. The concept of Pareto dominance is an extremely important part of multi-objective optimization, especially where some or all of the objectives and constraints are mutually conflicting. In such a case, there is no utopian point which leads to best answer for all objectives and constraints. The aim of multi-objective optimization in such a case is to find the best solutions (a Pareto or nondominated set) that are a group of solutions, when selecting any one of these solutions; on the one hand, the quality of one objective or constraint will be sacrificed, while on the other hand, the quality of at least one other objective or constraint will be boosted.

In general, for the generic optimization of any problem, the optimal Pareto could be defined as an ideal vector of which the components are separately attainable minima for all objective functions. So, the vector $f^{*}=\left(f_{1}^{*}, f_{2}^{*}, \ldots, f_{m}^{*}\right)$ where $f_{i}^{*}$ represents the scalar optimization solution of the $i^{\text {th }}$ objective function as $\min _{x \in X} f_{i}(x) \leq f_{i}^{*}$ for $i=1,2, \ldots, m$ at its relevant $x^{\prime}$.

Therefore, the ideal solution of a multi-objective optimization problem is the point (Pareto Optimal) in $X$ which determines $x^{*}$. Unfortunately, the Pareto optimization almost always gives a set of solutions, rather than a single solution. 
The Multi-Objective Genetic Algorithm (MOGA) optimizer in ANSYS DX incorporates global Pareto filters and leads to global Pareto front. To achieve this, a hybrid variant of controlled elitism concepts, NSGA-II, is used. The Pareto ranking scheme and objective and constraint handling are executed by a fast, non-dominated sorting method that is an order of magnitude faster than traditional Pareto ranking methods. To avoid premature convergence, ANSYS DX uses a "Percent Pareto" parameter, which lets users control the selection pressure (and consequently the elitism of the process).

\subsubsection{Optimization Model Settings}

79 Auto-defined CCD samples were specified for the 7 independent parameters. For each of these samples, an ANSYS Fluent simulation was run and the total heat loss for each point extracted. A Kriging response surface with variable variation type was then constructed for each of the output parameters and combined as necessary for the objectives and constraints. The default value for auto refinement of design points was chosen for the Kriging regression. Finally, the settings of the MOGA method: "Number of initial samples" and "Number of samples per Iteration" were set to 100, respectively, while the "Maximum allowable Pareto Percentage" was set to $80 \%$ and the "Maximum Number of Iterations" was set to 20 . This means that the optimization terminates its process when the resulting front of MOGA contains at least 80 points ( $80 \%$ of 100 as number of samples per iteration) or reaches the maximum number of iterations, whichever is reached sooner.

\section{Result and Discussion}

\subsection{Validation}

As mentioned before, previous researchers (Facão and Oliveira, 2011; Lai et al., 2013; Pye et al., 2003a, 2003b, 2003c; Pye, 2008; Sahoo et al., 2012 , 2013a) used the Boussinesq approximation in their modelling without verifying the accuracy of such an assumption. For instance, Pye (2008) mentioned that in some cases the density variation ratio $\Delta \rho / \rho=0.49$, which casts doubt on the accuracy of the CFD results. In the current paper, the temperature-dependent variation of density is solved using the incompressible ideal gas assumption. Especially because this was the first instance of its use in modeling cavity receivers to the best of the authors' knowledge, the accuracy of this assumption was first tested. The requirement for accuracy when modeling naturally convective flow is that the pressure variation should be insignificant when compared to temperature variation. The contours of static pressure and temperature in a sample case for a pipe outer surface temperature of $500 \mathrm{~K}$ with an ambient temperature of $300 \mathrm{~K}$ are displayed in Figure 6. It can be seen that the static pressure varies from -0.097 to 0.1 Pascal while temperature field varies from $290 \mathrm{~K}$ to $500 \mathrm{~K}$. This small variation in pressure as a result of the much larger temperature variation confirms that the incompressible ideal gas assumption is valid.

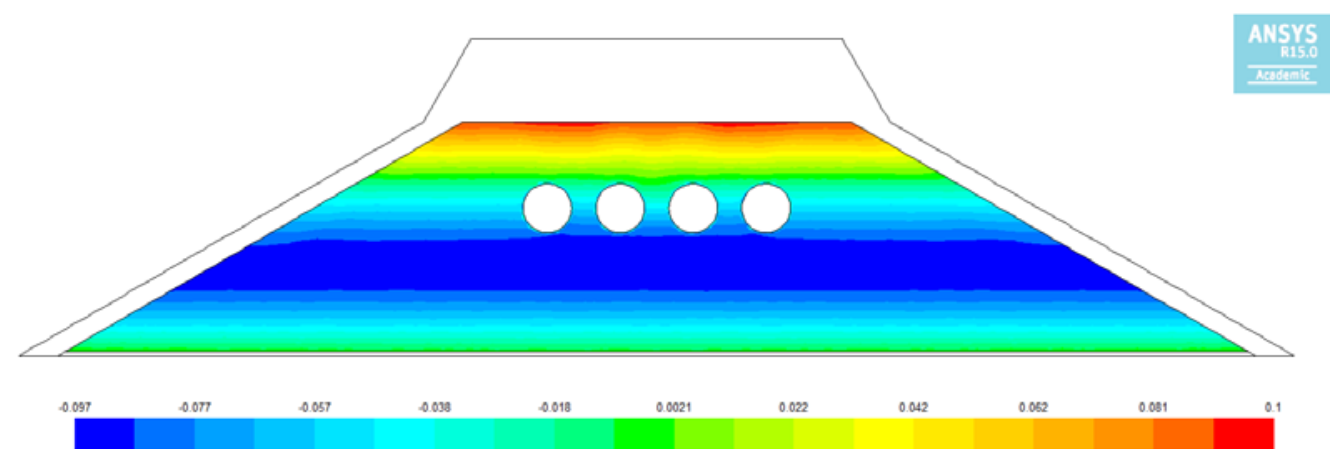

(a) 


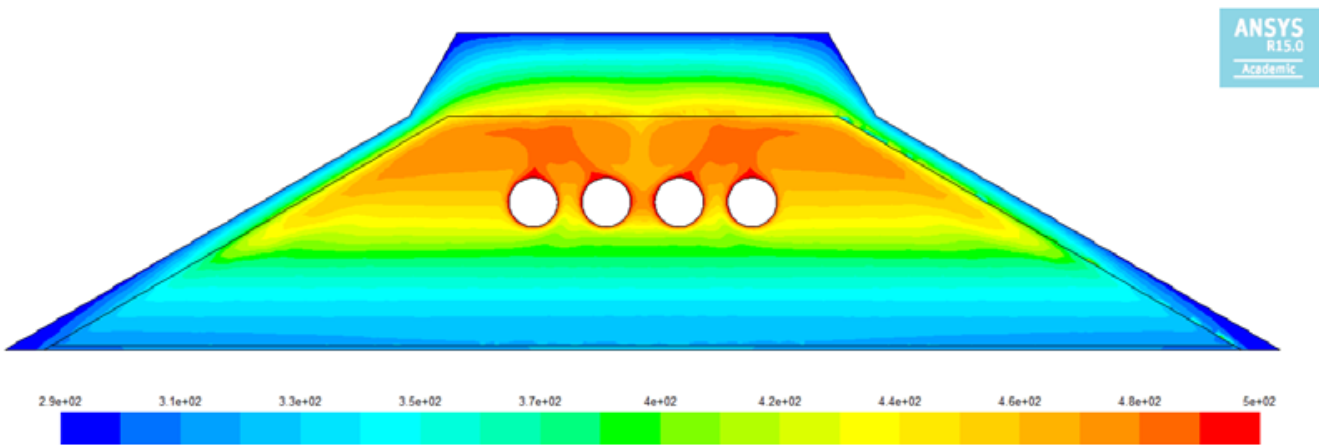

(b)

Figure 6: Contours inside cavity and in insulation for $500 \mathrm{~K}$ pipe outer surface temperature and $300 \mathrm{~K}$ ambient temperature for sample case geometry (a) Static pressure (b) Static temperature.

After getting assurance of the validation of the incompressible ideal gas assumption in the cavity receiver, a code-to-code validation case was done to survey the accuracy of the constant temperature assumption on the outer wall of pipe. The accuracy of this assumption which is widely used in other researchers' studies, has not been investigated to the best of the authors' knowledge to determine how far it deviates from a realistic situation (that has a non-uniform solar heat flux load on the outer surface of pipes). Of particular interest is how such an assumption would affect the calculation of heat losses from the external boundaries and how it would affect the fluid pattern in the cavity domain. Therefore, in this section of this paper, the accuracy of this assumption is investigated briefly.

To establish a reference, the non-uniform solar heat flux pattern on the pipes was calculated with the ray-tracing code, SolTrace (NREL, 2014). The results were mapped as a volumetric heat source on the pipes in a 3D conjugate heat transfer model of cavity receiver. More detailed information of the solar field, the Soltrace boundary conditions, ray number effects on the solution convergence of Soltrace, and the precise procedure for mapping non-uniform heat flux loads on pipes in 3D is described in Moghimi et al. (2015). Briefly, the solar LFR mirror field is based on the FRESDEMO plant (Bernhard et al., 2008) as displayed in Figure 7a. After simulating the optical performance at solar noon time with 1,000,000 rays (see sample rays in Figure 7a and 7b), the ray data were post-processed using a VBA code in Excel to provide a heat flux distribution. This was converted to a volumetric heat source by dividing by the thickness of the pipe (Moghimi et al., 2015). The cavity domain in the 3D model is a $1 \mathrm{~cm}$ extrusion of the 2D model in the z-direction that now includes the pipe thickness as well as the HTF inside the pipes. The HTF inside the pipes is assumed to be turbulent with fullydeveloped inlet profiles as defined by a User-Defined Function (UDF).

The mapping process to convert the SolTrace data to a volumetric heat source in ANSYS Fluent involves an interpolation file, a User-Defined Scalar, User-Defined Memory and a UDF (Moghimi et al., 2015). Once simulated, the 3D model determines how much of the solar irradiation is transferred to the HTF and how much is lost through the external boundaries involving all the heat transfer mechanisms. The latter can be directly compared to the current 2D case where a constant outside pipe surface temperature (uniform flux) is assumed.

Figure 8 displays radar plots of the non-uniform heat flux distributions on the two pipes in the right half of the cavity shown in Figure 2. Note the asymmetry in the rightmost distribution due to the presence of the oblique corner of the cavity insulation above this pipe. Figure 9 shows the 3D conjugate heat transfer domain in ANSYS Meshing (Figure 9a) and the mapped non-uniform solar flux as imposed on the pipes in ANSYS Fluent (Figure $9 b)$. The region with highly dense mesh includes HTF zones, pipe and surrounding of pipes in cavity receiver in Figure 9a. The correspondence between the distributions in Figure 8 and the contour colors in Figure 9b is clear.

A comparison between the temperature and velocity field in the cavity as obtained by the current 2D method and the comparative 3D method with a SolTrace distribution is depicted in Figure 10.

Figure 10 a depicts temperature contours overlaid with velocity vectors for the 3D with HTF model (at the mid cross-sectional plane) and the current 2D sample case. Good agreement is evident, especially in the natural convection plume formation. For the sample geometry it can be seen that natural convection would play a large 
role because of the convection of hot air from the pipe surfaces towards the upper insulation. Keep this fact in mind when the optimum results are presented later. As indicated in the figure caption, the minimum and maximum velocity magnitude values due to natural convection are very similar between the two simulations, further confirming the good agreement. The pipe outside surface temperature obtained in the 3D model is compared to the uniform temperature specified in the 2D case in the insert in Figure 10a. The effect of the nonuniform heat flux distribution clearly results in a similarly shaped non-uniform temperature distribution with deviations on the order of 2 to $3 \mathrm{~K}$. The average outside surface temperature of $500 \mathrm{~K}$ for the 3D case (to be comparable with the specified 2D value of 500K) was achieved by a trial-and-error procedure by varying the bulk inlet HTF temperature with a converged value of 492K. The heat loss values (indicated in Figure 10a and $10 \mathrm{~b}$ and also summarized in Table 5) confirm that there is excellent agreement between the two models with the total heat loss differing by less than $1 \%$. This implies that at these temperatures, the uneven distribution in heat flux does not play a significant role in the thermal performance of the cavity and that the 2D setup can be used for optimization purposes. Note that the 3D heat loss values were scaled by 200 for a direct comparison (2x because of the half symmetry and 100x to compensate for the $1 \mathrm{~cm}$ length of the pipes because $2 \mathrm{D}$ results are reported per meter or per $100 \mathrm{~cm}$.)
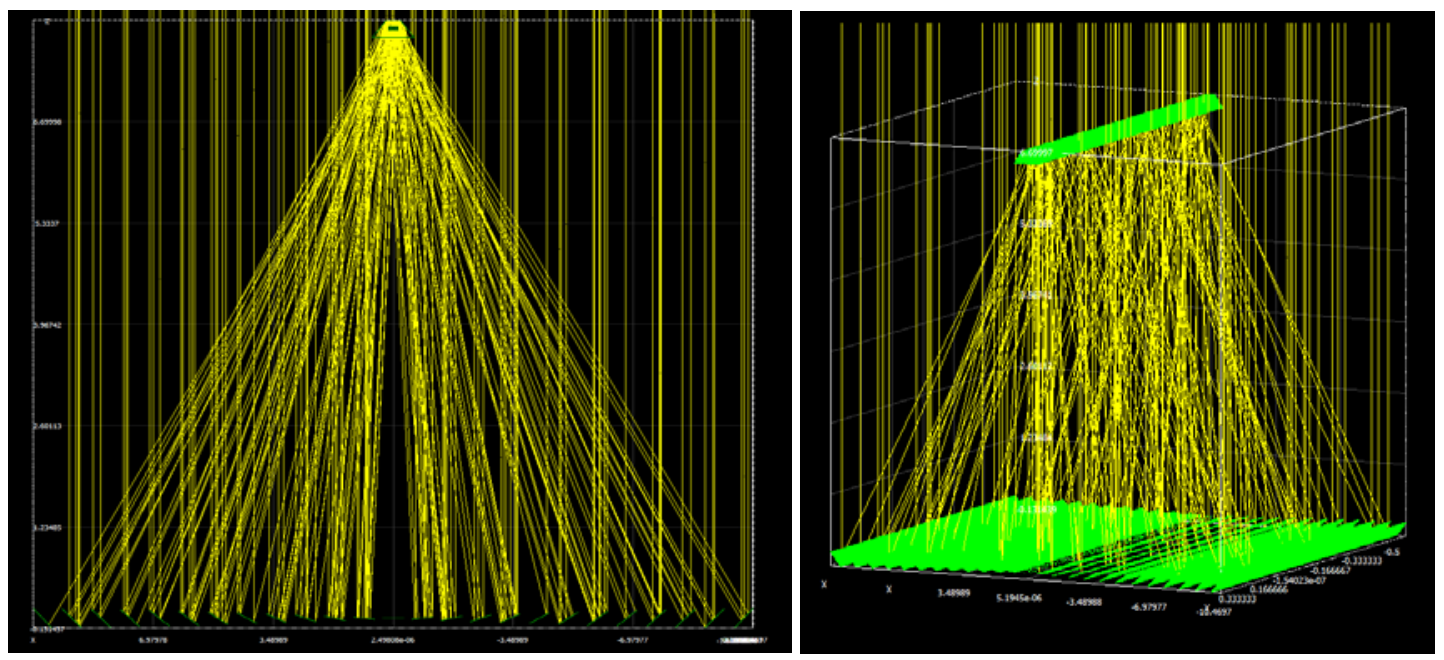

a)

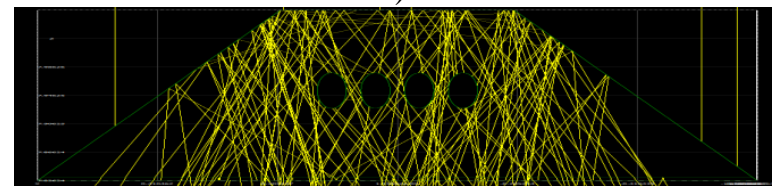

b)

Figure 7: Ray Tracing for the sample Linear Fresnel solar field in SolTrace a) Entire optical domain (front and isometric view), b) Zoomed view of cavity receiver with four pipes 


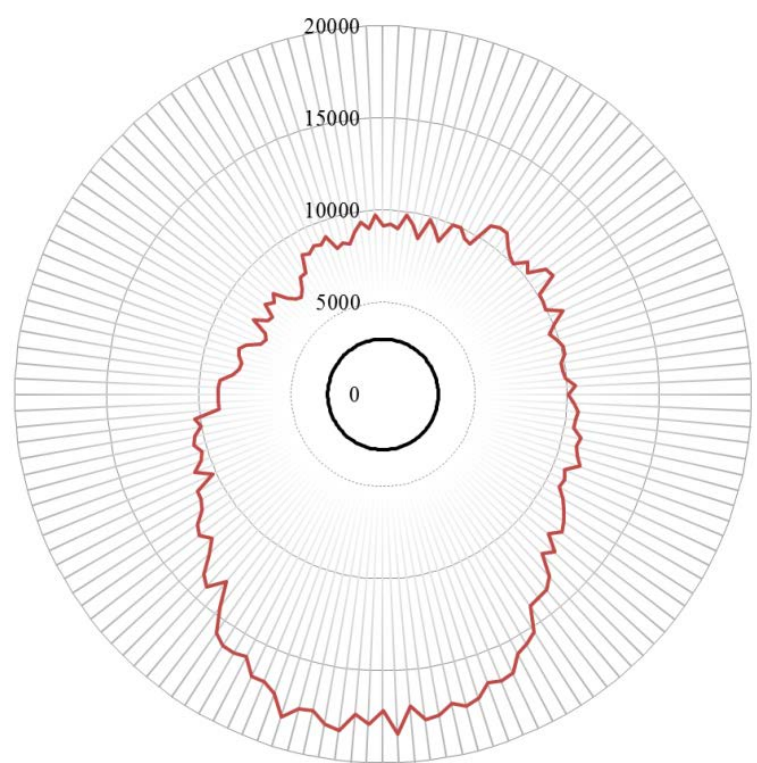

a)

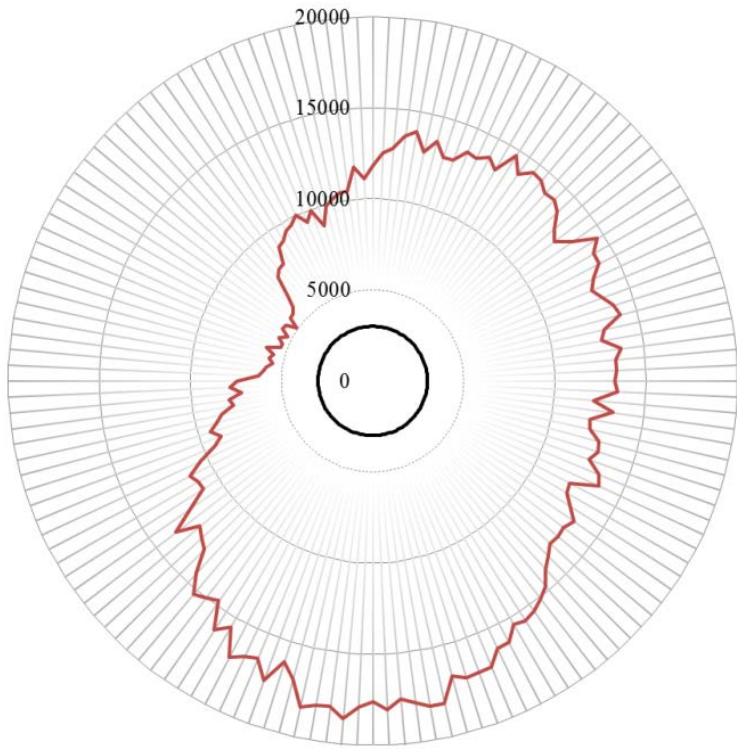

b)

Figure 8: Non-uniform solar heat flux distribution as calculated by SolTrace on a) $3^{\text {rd }}$ pipe from left, b) $4^{\text {th }}$ pipe from left in $\mathrm{W} / \mathrm{m}^{2}$.

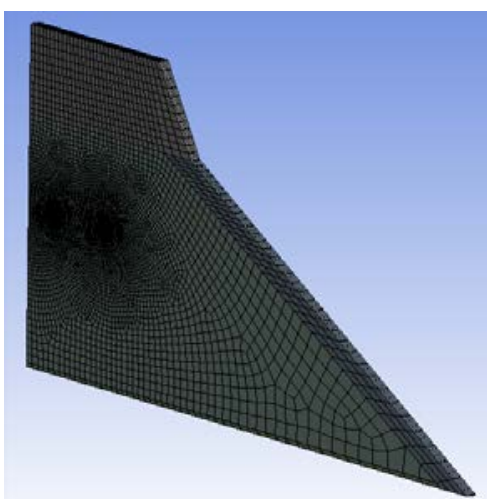

a)

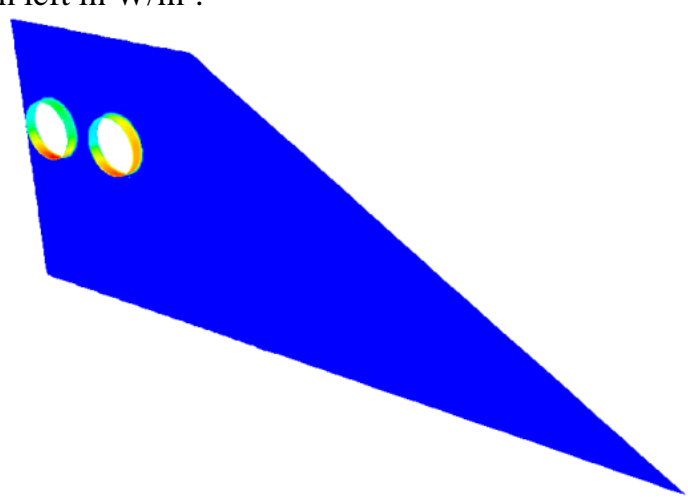

b)

Figure 9: a) 3D CFD cavity domain with mesh, b) Mapped non-uniform solar heat flux $\left[\mathrm{W} / \mathrm{m}^{3}\right]$ as volumetric heat load on the pipes of cavity receiver in ANSYS Fluent 


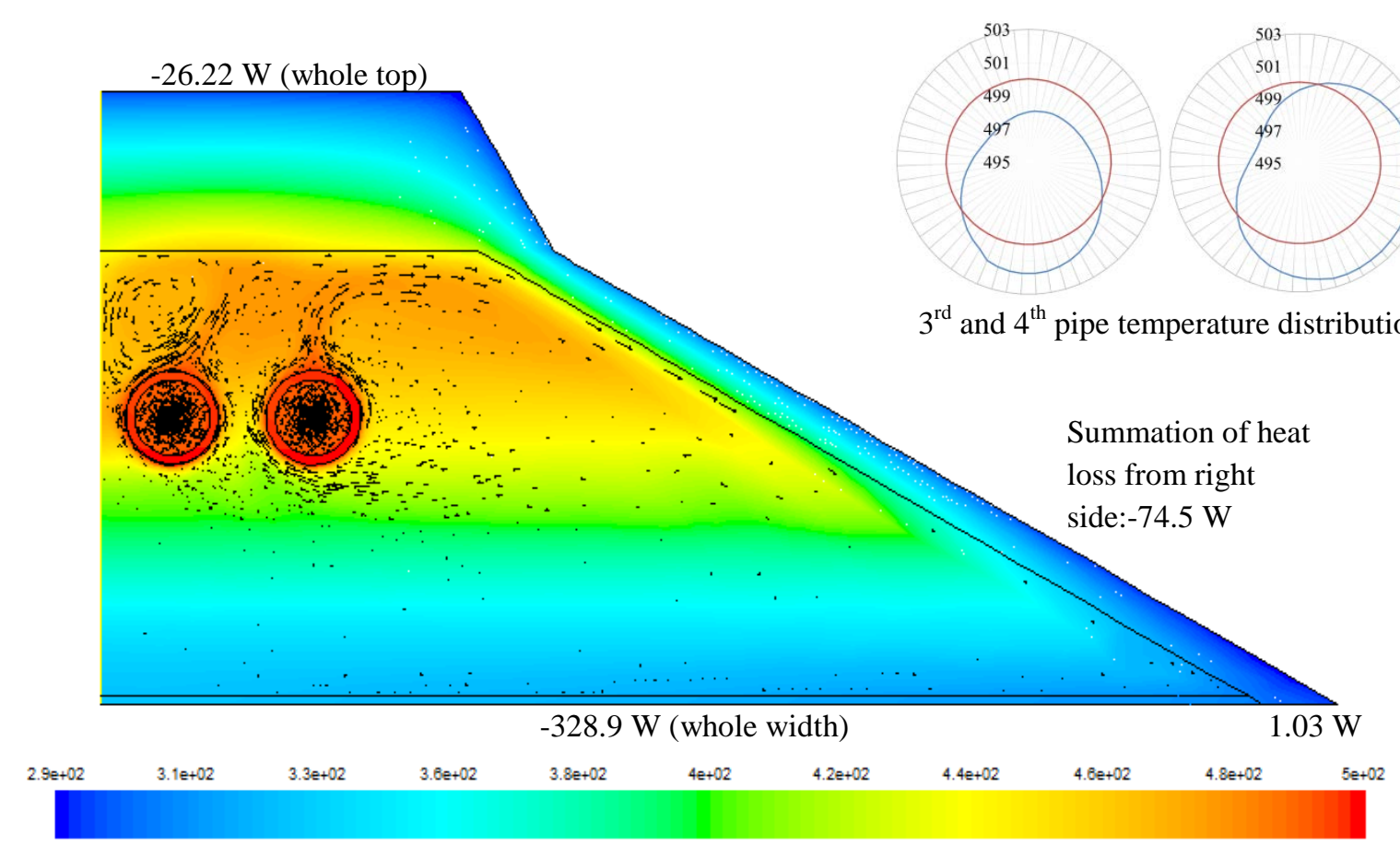

a) Maximum velocity (mid plane of 3D domain) $=0.15 \mathrm{~m} / \mathrm{s}$, minimum velocity $=3.6 \mathrm{e}-5 \mathrm{~m} / \mathrm{s}$. Insert showing deviation of the non-uniform (blue) temperature distribution from a uniform (red) distribution around the two pipes shown

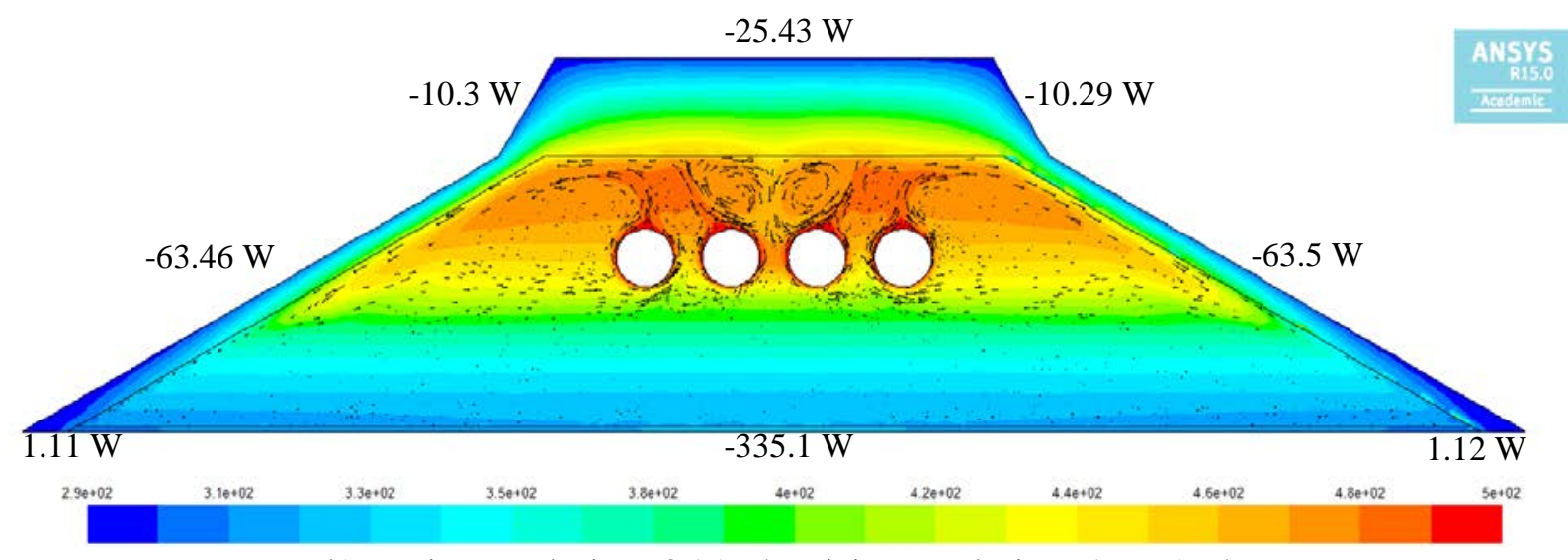

b) Maximum velocity $=0.15 \mathrm{~m} / \mathrm{s}$, minimum velocity $=1.9 \mathrm{e}-5 \mathrm{~m} / \mathrm{s}$

Figure 10: Temperature contours $[\mathrm{K}]$ overlaid with velocity vectors and heat losses and gains from external boundaries; a) on the mid-plane of 3D case, b) 2D sample case

\begin{tabular}{|c|c|c|}
\hline \multicolumn{2}{|c|}{ Table 5: Heat loss comparison from external boundaries for 2D and 3D cases (W) } \\
\hline Surfaces & 2D sample case (per meter) & 3D case (* 200) \\
\hline glass & -335.1 & -328.9 \\
\hline $\begin{array}{c}\text { Insulation bottom surfaces } \\
\text { (right and left sides) }\end{array}$ & 2.232 & 2.062 \\
\hline $\begin{array}{c}\text { Insulation side surfaces } \\
\text { (right and left sides) }\end{array}$ & -147.6 & -149.1 \\
\hline Insulation top surface & -25.46 & -26.22 \\
\hline Total heat loss & -505.9 & -502.2 \\
\hline
\end{tabular}

To conclude the validation section, a comparison is made with the correlation for heat loss provided by Flores Larsen et al. (2012). In this validation comparison of published experimental Heat Loss Coefficient power-law 
correlations with the CFD model results was done. The Heat Loss Coefficient $\left(U_{L}\right)$ is one of the thermal performance indices of a cavity receiver. According to Flores Larsen et al. (2012) $\mathrm{U}_{\mathrm{L}}$ is defined as:

$U_{L}=\frac{Q_{\text {loss }}}{A_{p}\left(T_{p}-T_{a}\right)}$

According to Flores Larsen et al. (2012), the empirical correlation of $U_{L}$ for a cavity receiver should follow a power-law relation with respect to the area of all pipes, $A_{p}$, and the difference between the average of all the pipes' outside wall temperatures at their lowest points facing the incoming irradiation, $T_{p}$, and the ambient temperature, $T_{a}$, i.e.,

$U_{L}=x\left(T_{p}-T_{a}\right)^{y}$

where $x$ and $y$ are constants. Such a power law concept is motivated by the observation that the losses would significantly increase with temperature. A summary of power law correlations found in literature for a single glass cover trapezoidal cavity is presented in Table 6 and compared to the current sample case solved for seven different pipe outside temperatures (350, 375, 400, 425, 450, 475 and $500 \mathrm{~K}$ ). The corresponding Heat Loss Coefficient is plotted in Figure 11 against the temperature difference with a power law fitted. As similar result for $x$ and $y$ in equation 24 is found as by other researchers (see Table 6), but it can be seen from the simulated values that a power law fit is not necessarily the best fit. Considering, e.g., a linear, exponential, quadratic or cubic curve fit, regression coefficient values of $0.9944,0.9991,0.9995$ and 1, respectively, are obtained. The latter's good performance is interesting, because it could have a physical reason. If most of the energy is dissipated through radiation (as was confirmed by the relative heat loss values in Figure 10), then the heat loss would be expected to be a quartic function of the temperatures: $Q_{\text {loss }}=f\left(T_{p}^{4}-T_{a}^{4}\right)$, and thus based on equation 23, the Heat Loss Coefficient will be a cubic function of pipe and ambient temperature $U_{L}=f\left(T_{p}^{3}, T_{a}^{3}\right.$,combination oflower orders $)$. A cubic polynomial fit is also shown in Figure 11 and should be considered for future comparison.

\begin{tabular}{|c|c|c|c|}
\hline \multirow{2}{*}{ Researchers } & Power law relation & $\begin{array}{c}\text { Regression } \\
\text { coefficient }\end{array}$ & Restriction \\
\hline This paper & $U_{L}=0.3341\left(T_{p}-T_{a}\right)^{0.4443}$ & 0.9703 & $350 \mathrm{~K}<T_{p}<500 \mathrm{~K}$ \\
\hline $\begin{array}{c}\text { Florens Larsen } \\
\text { et al. (2012) }\end{array}$ & $U_{L}=0.357\left(T_{p}-T_{a}\right)^{0.5184}$ & 0.8862 & $110^{\circ} \mathrm{C}<T_{p}<285^{\circ} \mathrm{C}$ \\
\hline \multirow{4}{*}{$\begin{array}{c}\text { Singh et al. } \\
\text { (2010) }\end{array}$} & $U_{L}=0.3521\left(T_{a b s}\right)^{0.6076}$ & 0.9932 & $\begin{array}{c}\text { In Singh et al }(2010) T_{a b s} \text { is absorber } \\
\text { temperature in }{ }^{\circ} \mathrm{C} \text { and the valid range is } \\
50^{\circ} \mathrm{C} \leq T_{a b s} \leq 250^{\circ} \mathrm{C} \text {.This relation is for a } \\
\text { rectangular pipe absorber covered with ordinary } \\
\text { black paint. }\end{array}$ \\
\cline { 2 - 4 } & $U_{L}=0.2687\left(T_{a b s}\right)^{0.5858}$ & 0.9887 & $\begin{array}{c}\text { This relation is for a rectangular pipe absorber } \\
\text { covered with a selective surface coating. }\end{array}$ \\
\cline { 2 - 5 } & $U_{L}=0.5805\left(T_{a b s}\right)^{0.4903}$ & ---- & $\begin{array}{c}\text { This relation is for a round pipe absorber } \\
\text { covered with ordinary black paint. }\end{array}$ \\
\cline { 2 - 5 } & $U_{L}=0.5637\left(T_{a b s}\right)^{0.4456}$ & ---- & $\begin{array}{c}\text { This relation is for a round pipe absorber } \\
\text { covered with a selective surface coating. }\end{array}$ \\
\hline
\end{tabular}




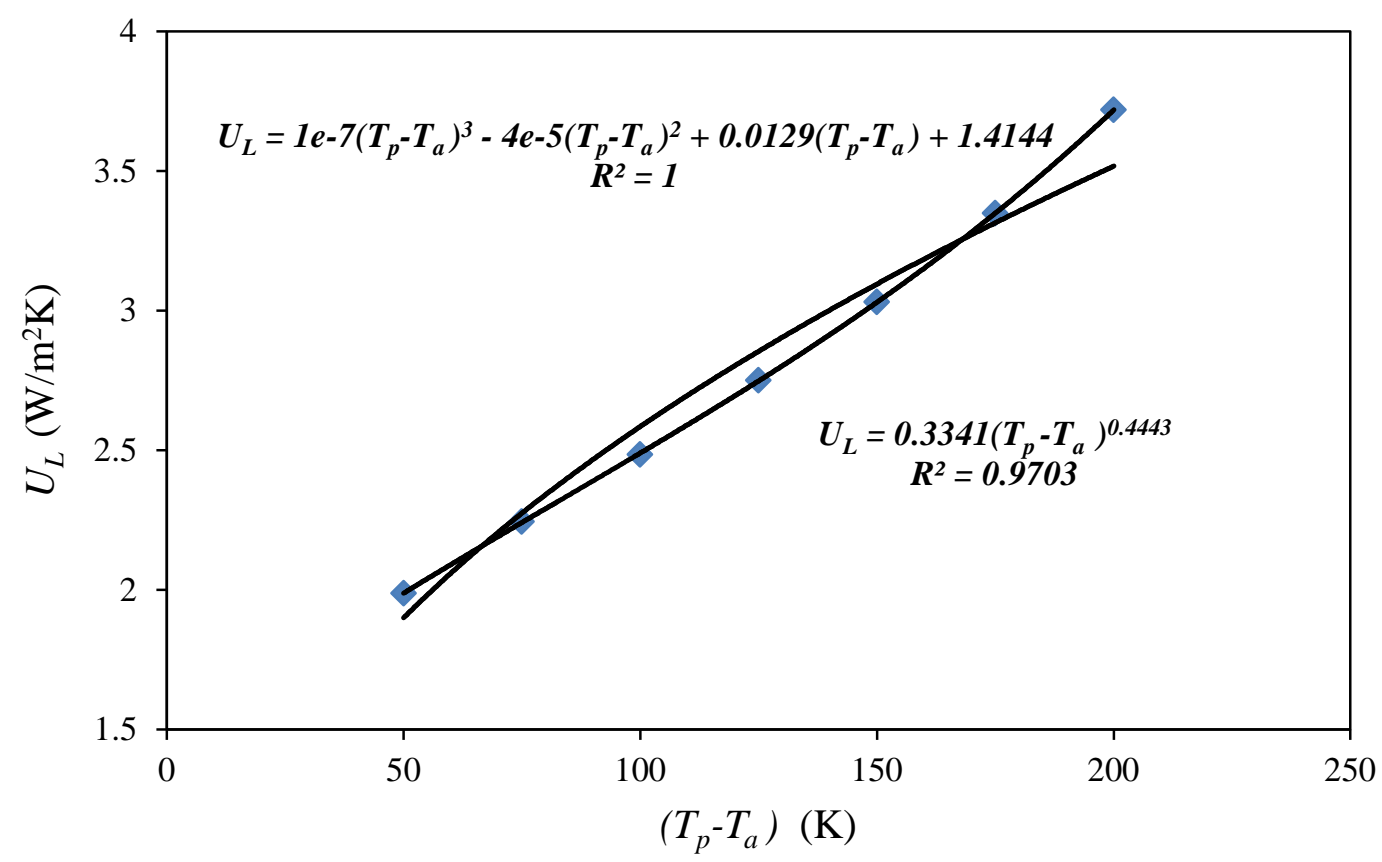

Figure 11: Heat loss coefficient variation and power law and cubic polynomial fits for sample case geometry

\subsection{Optimization Results}

The optimization on the combined Kriging response surfaces converged after 883 iterations when considering a lower objective importance for "area" and a higher objective importance for "half of heat loss". The local sensitivity of objectives in the response surface for specific values of the independent parameters is shown in Figure 12. In addition since it is impossible from a physical viewpoint to display a surface in more than three dimensions, four 3D response surfaces were prepared which show the effect of two independent parameters on one optimization objective. These figures are depicted in Figure 13a to Figure 13d. The information of the local sensitivity graph of Figure 12 was used to select the most sensitive parameters for the response surface plots.

Considering Figure 13a, for example, it shows that a smaller pipe pitch (parameter $m$ ) and a smaller distance between the pipes and the top insulation (parameter $d$ ) would result in a less negative (i.e., lower) heat loss. I.e., the response surface indicates that the optimizer should strive to move the pipe closer together and to bunch them up against the top insulation. This makes sense physically for three reasons. First, a smaller gap would limit the natural convective cooling between pipes because of the increased flow resistance (the Rayleigh number is related to the third power of the distance between hot and cold sources), second, the closer proximity to the top insulation would limit the formation of buoyant plumes that would impact the insulation, and, third, a smaller $d$ and $m$ would reduce the view factor from the pipes to the rest of the cavity, thereby reducing reradiation. There is however a limit to how realistic this effect is, and this highlights a shortcoming of the constant temperature (uniform heat flux) assumption currently being employed. This shortcoming centers around the fact that a setup with pipes close to the top (currently assumed to be highly reflective) wall would not receive a significant amount of solar irradiation on the upper half because of the shadowing and block effect of adjacent pipes, implying that the uniform assumption would degrade in its applicability. This means that to truly drive to an optimum design with total freedom of all the design parameters, the phased approach that includes both the optics and a 3D thermal solution as briefly outlined in the validation section, would be required. This is a topic of future work.

The response surface in Figure 13b provides the expected result that thicker insulation all around the cavity would limit the heat loss while Figure 13d shows expectedly that a thinner top insulation thickness and lower depth cavity would reduce the side wind area. Interestingly, Figure 13c indicates that a deep cavity with a side angle close to the lower limit of $30^{\circ}$ would be optimal in terms of heat loss. This makes sense as the hottest part 
of the cavity is the top wall, and driving these two parameters in such a way would reduce the length and hence the radiating area of this top surface.

Values for three utopian points were automatically selected by ANSYS DesignXplorer among all feasible Pareto optimal cases. Before these are discussed, it must be stressed that the above observations are based on a single specified pipe temperature where there is a unique combination between the three heat transfer mechanisms. Typically in an LFR plant, there is a steady rise in pipe temperature with the overall rise depending on HTF type and routing of pipes through the plant. It is conceivable that the optimal cavity layout could be different for different parts of the plant depending on the local temperature. As an attempt to investigate this effect, the optimization process was repeated for one other temperature, namely, 350K.

The utopian points suggested for both temperatures are summarized in Table 7. As expected, the heat loss values for the $500 \mathrm{~K}$ temperature case are nearly an order of magnitude larger than that for a $350 \mathrm{~K}$ pipe temperature. If the heat loss is mainly radiation driven, then $\left(500^{4}-305^{4}\right) /\left(350^{4}-305^{4}\right) \sim 8.5$. The actual ratios in Table 7 are approximately 7. As shown in the detail CFD results below (Figure 14) for one of the utopian points, the reradiation loss through the glass cover is reduced by a factor of 8.1 when lowering the pipe temperature from $500 \mathrm{~K}$ to $350 \mathrm{~K}$. As expected, the wind area objective is mainly unchanged by the thermal aspects driving the heat loss objective. Interestingly, expect for top insulation angle $\theta_{2}$, all the other parameters have similar values for the utopian points of the two specified pipe temperatures. Not having evaluated other temperatures, this observation cannot be generalized, but it would seem as if the heat loss mechanisms are in a similar balance for the two temperatures considered.

Because of the similar performance of all the utopian points and the similarity of their design, candidate 3 for the $500 \mathrm{~K}$ was arbitrarily selected for further investigation. Because of manufacturing limitations, the parameters of this candidate were rounded to feasible values and the CFD simulation repeated (summary results also listed in Table 7.)

Finally, the optimum values obtained in the response surface optimization are based on the Kriging response surfaces, i.e., predictions based on a surrogate model. The optimal set of parameters was fed into the CFD model to determine actual or verified results (also listed for the objectives in Table 7.)

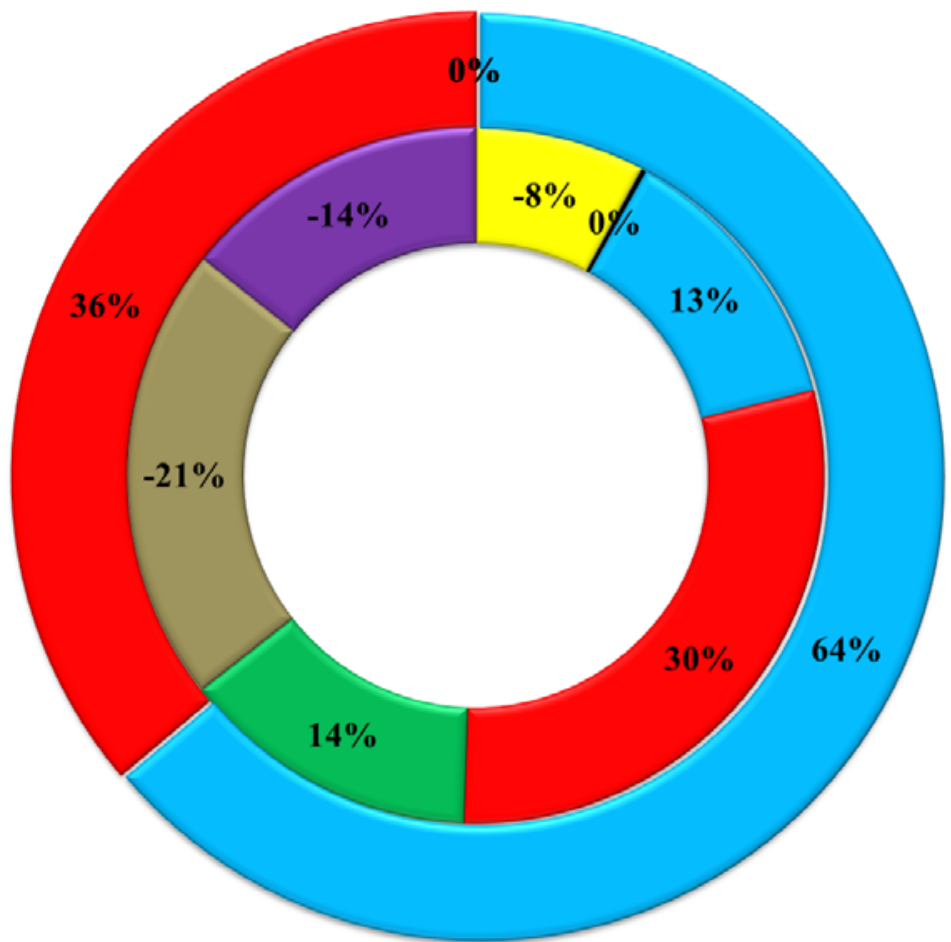

\title{
Local Sensitivity of "half of heat loss" (inner ring) and "area" (outer ring)
}

\author{
$\square$ P1 - Thetal_Cavity angle (degree) \\ घP2 - Theta2_Insulation angle (degree) \\ 圆4 - e_Cavity depth (mm) \\ 曰P5 - f_Top insulation thickness (mm) \\ ๑ P6 - a_Side insulation (mm) \\ $\square$ P8 - d_Distance from top side (mm) \\ घP11 - m_Pipe pitch (mm)
}

Figure 12: Local sensitivity of two objectives ("half of heat loss” and "area”) versus independent parameters at a local point in the total response surface model. The independent parameter values at the local point are 
$e=225 \mathrm{~mm}, f=52.5 \mathrm{~mm}, a=55 \mathrm{~mm}, d=67.5 \mathrm{~mm}, m=63.5 \mathrm{~mm}, \theta_{1}=30^{\circ}$ and $\theta_{2}=67.5^{\circ}$.

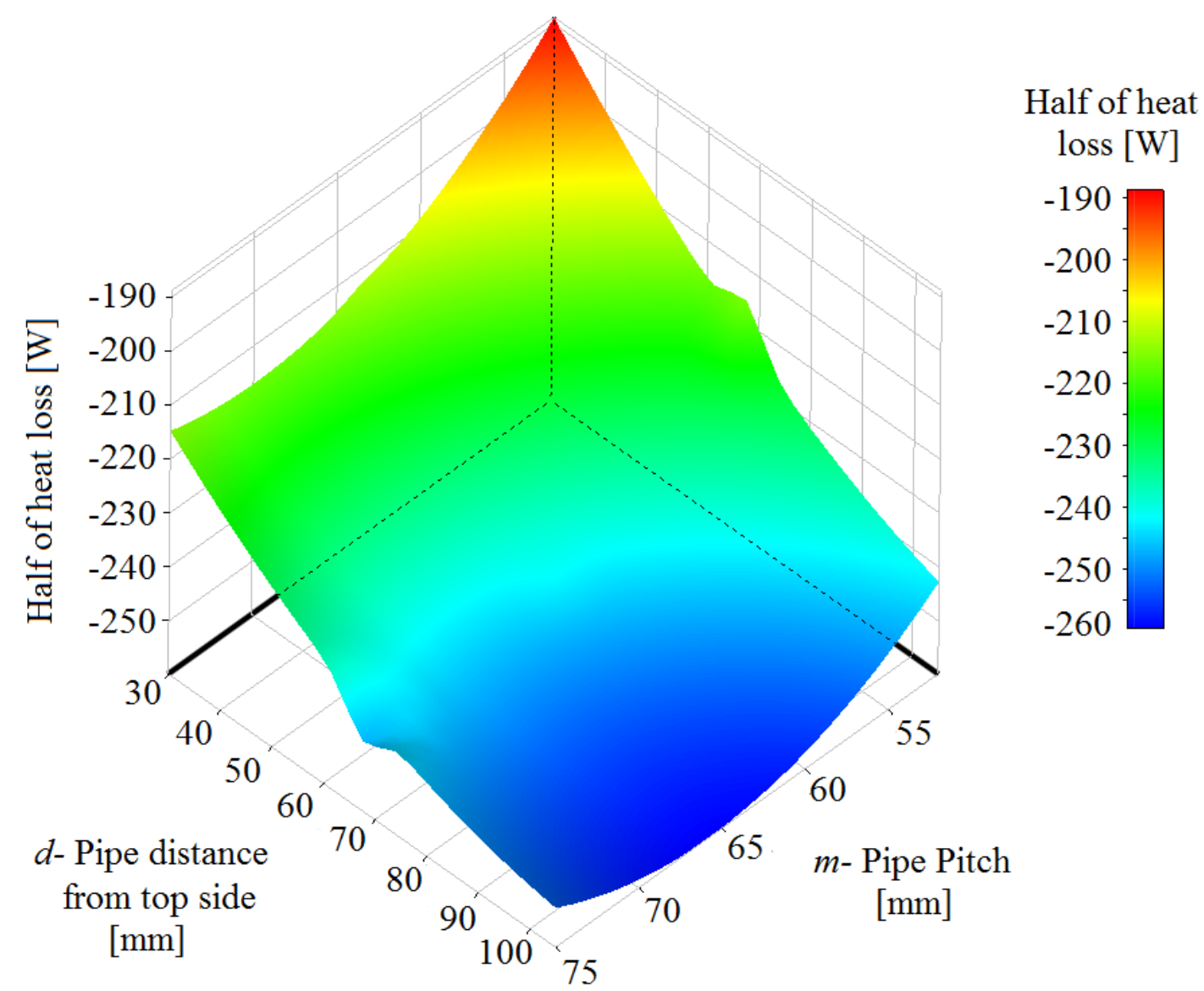

a) Response surface of "half of heat loss" (z-axis) vs. $m$-pipe pitch (y-axis) and $d$-pipe distance from cavity top wall (x-axis) 


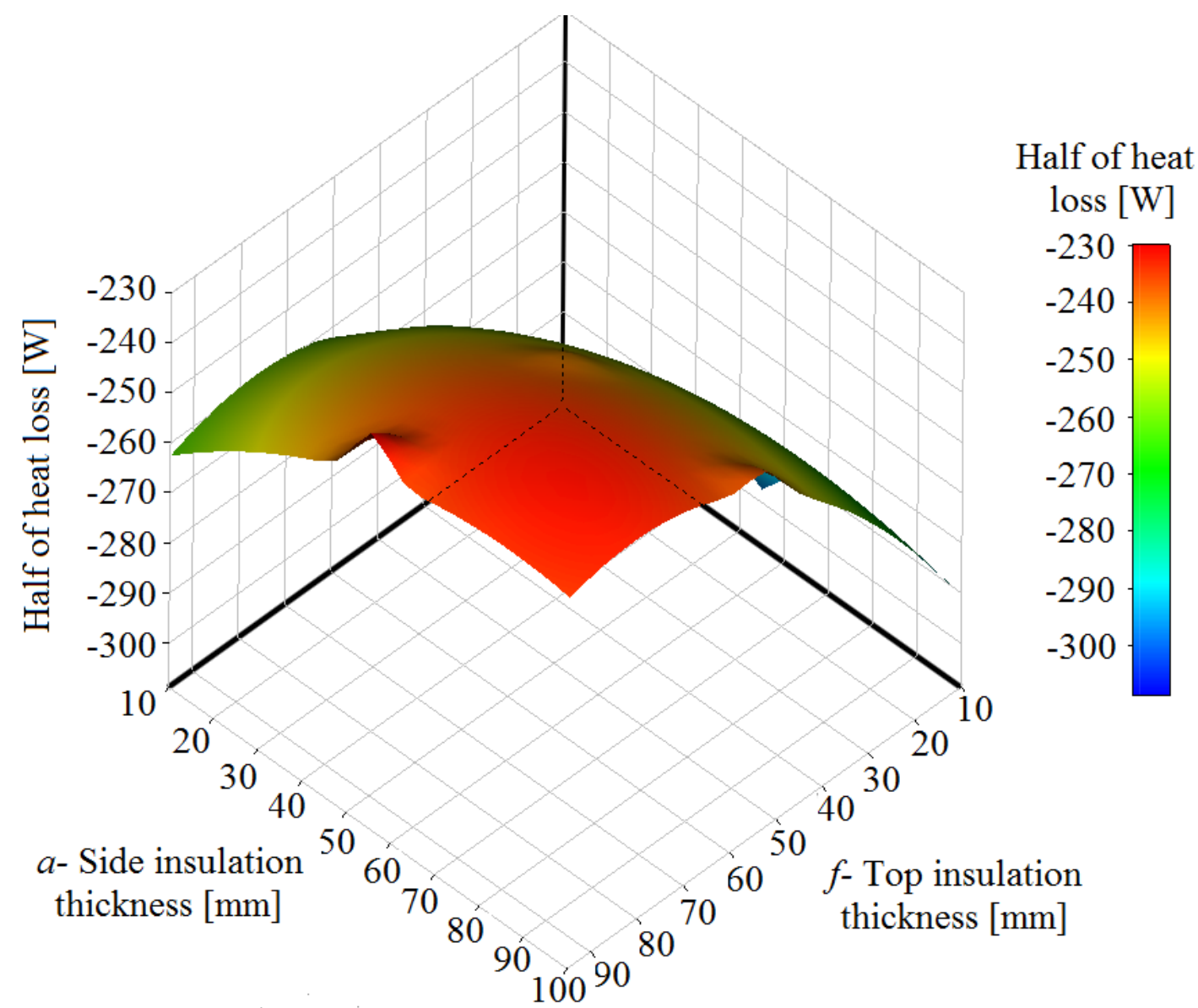

b) Response surface of "half of heat loss" (z-axis) vs. $f$-top insulation thickness (y-axis) and $a$-side insulation thickness (x-axis) 


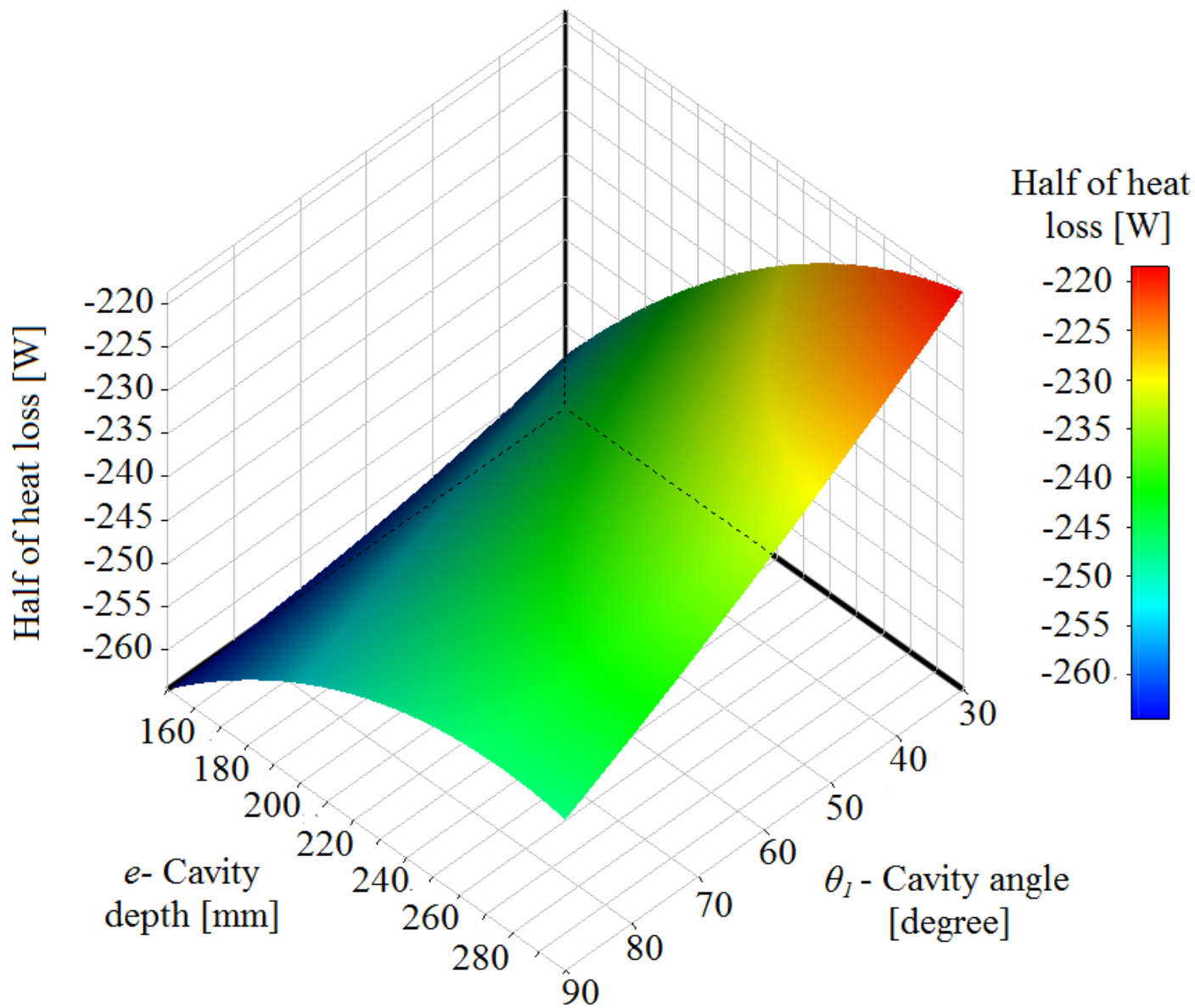

c) Response surface of "half of heat loss" (z-axis) vs. $\theta_{1}$-cavity angle (y-axis) and $e$-cavity depth (x-axis)

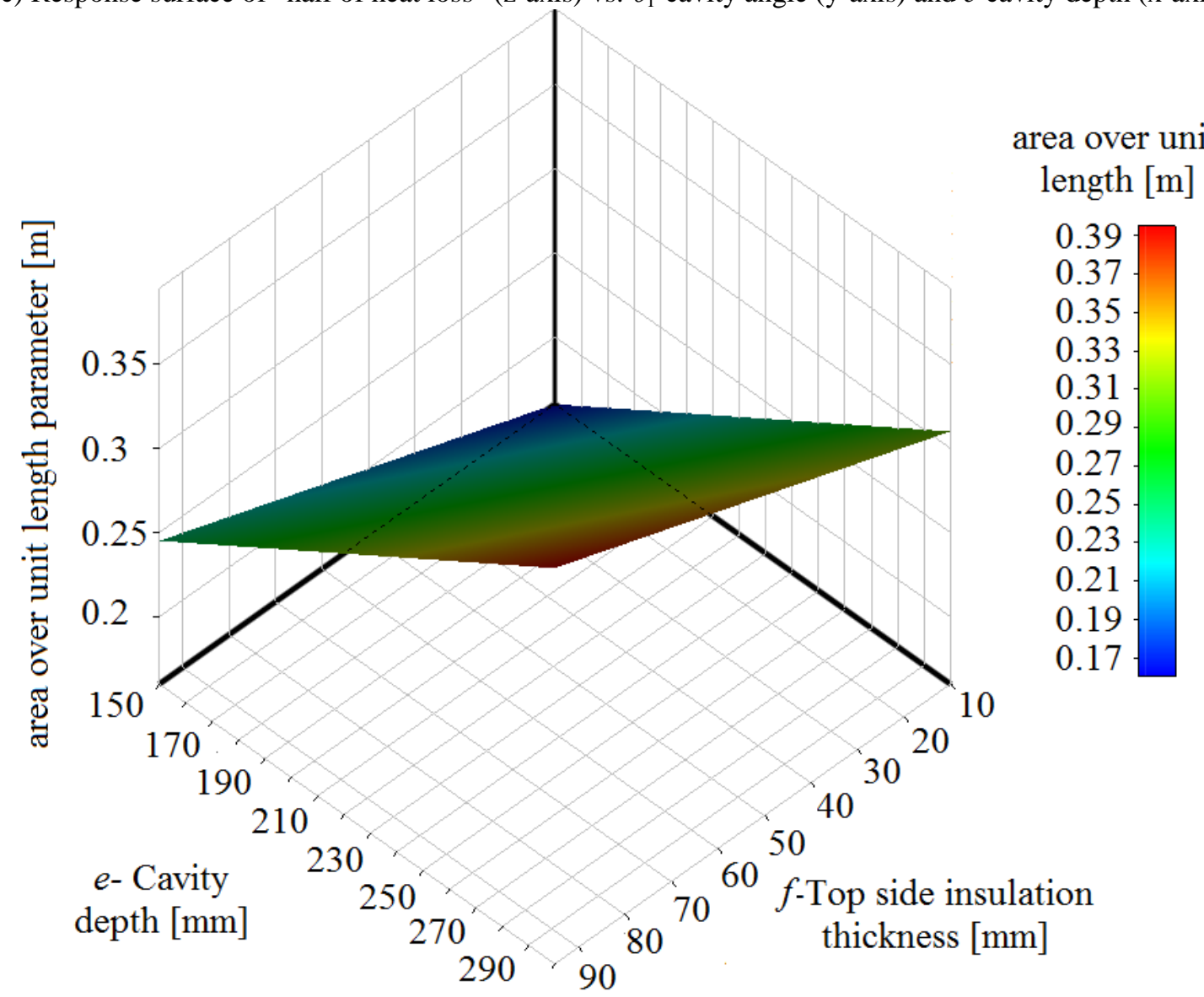


d) Response surface of "area” over unit length parameter (z-axis) vs. $f$-top insulation thickness (y-axis) and e-cavity depth (x-axis)

Figure 13: 3D response surfaces of each objective versus independent parameters

\begin{tabular}{|c|c|c|c|c|c|c|c|}
\hline \multirow{2}{*}{$\begin{array}{l}\text { Parameters } \\
\text { (unit) }\end{array}$} & \multicolumn{3}{|c|}{ Pipe surface temperature $500 \mathrm{~K}$} & \multicolumn{3}{|c|}{ Pipe surface temperature 350K } & \multirow{2}{*}{$\begin{array}{c}\text { feasible } \\
\text { optimum } \\
\text { case }\end{array}$} \\
\hline & 1st candidate & 2nd candidate & $\begin{array}{c}\text { 3rd } \\
\text { candidate }\end{array}$ & 1st candidate & $\begin{array}{c}\text { 2nd } \\
\text { candidate }\end{array}$ & $\begin{array}{c}\text { 3rd } \\
\text { candidate }\end{array}$ & \\
\hline$a(\mathrm{~mm})$ & 70.481 & 56.52 & 69.484 & 69.346 & 77.565 & 81.098 & 69 \\
\hline$f(\mathrm{~mm})$ & 55.985 & 61.325 & 64.296 & 62.316 & 66.224 & 67.101 & 64 \\
\hline$e(\mathrm{~mm})$ & 150.26 & 168.46 & 152.74 & 165.81 & 197.1 & 154.94 & 153 \\
\hline$m(\mathrm{~mm})$ & 52.702 & 52.691 & 52.342 & 52.086 & 52.245 & 52.023 & 52 \\
\hline$d_{(\mathrm{mm})}$ & 30.201 & 30.094 & 30.432 & 30.11 & 30.379 & 30.64 & 30 \\
\hline$\theta_{2}\left(^{\circ}\right)$ & 53.672 & 54.255 & 53.801 & 75.14 & 75.005 & 74.822 & 54 \\
\hline$\theta_{1}\left({ }^{\circ}\right)$ & 30.19 & 31.001 & 37.0784 & 31.006 & 30.308 & 31.825 & 37 \\
\hline $\begin{array}{l}\text { Optimization } \\
\text { predicted } \\
\text { value for } \\
\text { half of heat } \\
\text { loss (W) } \\
\text { [verified } \\
\text { value] } \\
\end{array}$ & $\begin{array}{c}-192.5 \\
{[-190.47]}\end{array}$ & $\begin{array}{c}-186.95 \\
{[-187.31]}\end{array}$ & $\begin{array}{c}-190.82 \\
{[-187.11]}\end{array}$ & $\begin{array}{c}-28.883 \\
{[-28.519]}\end{array}$ & $\begin{array}{c}-26.921 \\
{[-27.145]}\end{array}$ & $\begin{array}{c}-29.547 \\
{[-28.539]}\end{array}$ & ---- \\
\hline $\begin{array}{l}\text { Optimization } \\
\text { predicted } \\
\text { value for } \\
\text { area (mm) } \\
\text { (Wind } \\
\text { area/unit } \\
\text { length) } \\
\text { [verified } \\
\text { value] }\end{array}$ & $\begin{array}{c}0.20625 \\
{[0.20625]}\end{array}$ & $\begin{array}{c}0.22979 \\
{[0.22979]}\end{array}$ & $\begin{array}{c}0.21703 \\
{[0.21703]}\end{array}$ & $\begin{array}{c}0.22813 \\
{[0.22813]}\end{array}$ & $\begin{array}{c}0.26333 \\
{[0.26333]}\end{array}$ & $\begin{array}{c}0.22205 \\
{[0.22205]}\end{array}$ & ---- \\
\hline
\end{tabular}

The power of the MOGA algorithm is evident when evaluating the utopian designs in a similar manner that the individual response surfaces in Figure 13 were evaluated. In that discussion, the sensitivity of single objectives with respect to certain design parameters was discussed. When considering both objectives, compromises are made. The results in Table 7 confirm that the pipe spacing $(m)$ and location $(d)$ and cavity angle $\left(\theta_{1}\right)$ tend towards their lower bound (as listed in Table 1) because of them not influencing the wind area, i.e., they are driven by a single objective, the heat loss value (also confirmed by their respective sensitivities in Figure 12). The cavity depth $(e)$ is driven to close to its lowest level for most of the utopian points because of its large influence on the other objective, the wind area, even though making it larger would limit the heat loss. This compromise is explained in the sensitivity wheel of Figure 12 where the parameter $e$ was shown to be responsible for $64 \%$ of the wind area's change and only $13 \%$ of the heat loss's change. The other parameters (the insulation thickness parameters ( $a$ and $f$ ), and top insulation angle $\left(\theta_{2}\right)$ ) do not exhibit a clear trend, with different combinations of them leading to similar performance. This could present an opportunity to link other objectives to the optimization process to exploit differences between these designs (e.g., economic decisions, material or manufacturing cost, etc.). This is a topic of future work.

An interesting alternative to the current implementation would be to investigate the number of pipes and whether locating the pipes partially into the insulation would prove beneficial. For such a study, the optical performance must be included to account for the trade-off between absorbed radiation and re-radiation losses would have to be made. 


\subsection{Final CFD Results}

Detailed CFD results for the feasible optimum case with two specified pipe wall temperatures are given in Figure 14 in the form of temperature contours overlaid with velocity vectors due to natural convection. The stratification of temperature with negative slope leads to low velocities in the lower part of the receiver while high-velocity vectors can be seen in the regions close to the pipes. This proves the claim that in the lower region of cavity receiver, radiation and conduction heat transfer mechanisms dominate. These observations hold true for both temperatures considered. The heat loss contributions from the various external surfaces are also listed in Figure 14 with the re-radiation through the glass cover being dominant.

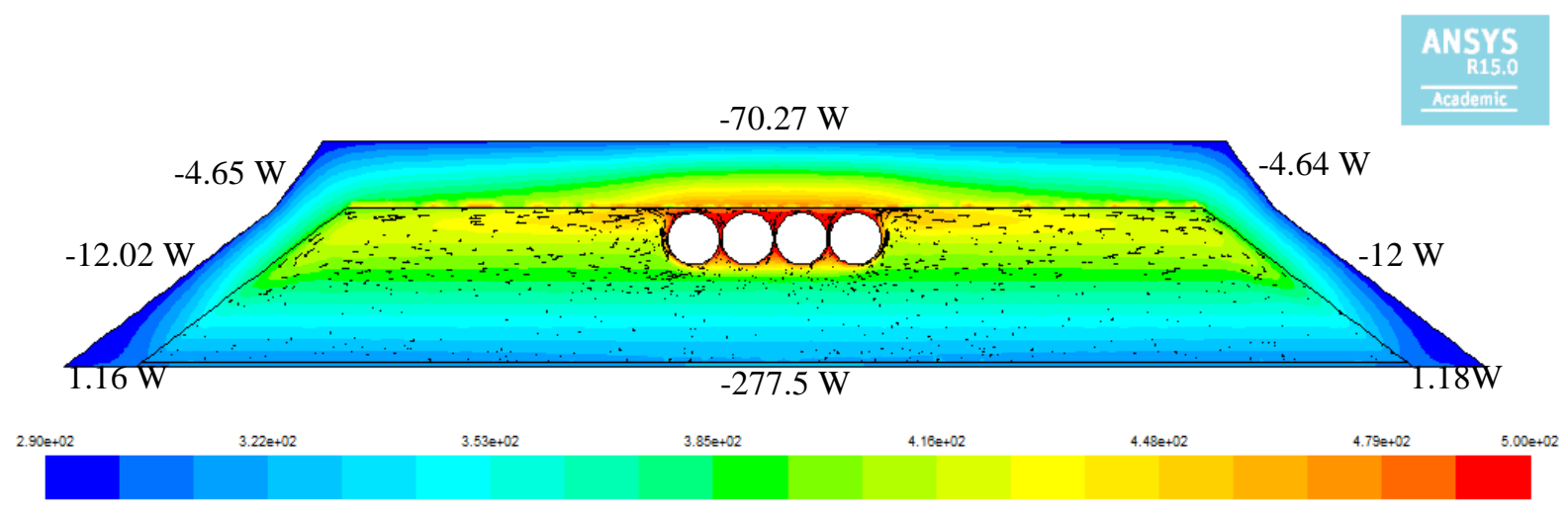

(a) Maximum velocity $0.134 \mathrm{~m} / \mathrm{s}$ and minimum velocity $2.69 \mathrm{E}-5 \mathrm{~m} / \mathrm{s}$.

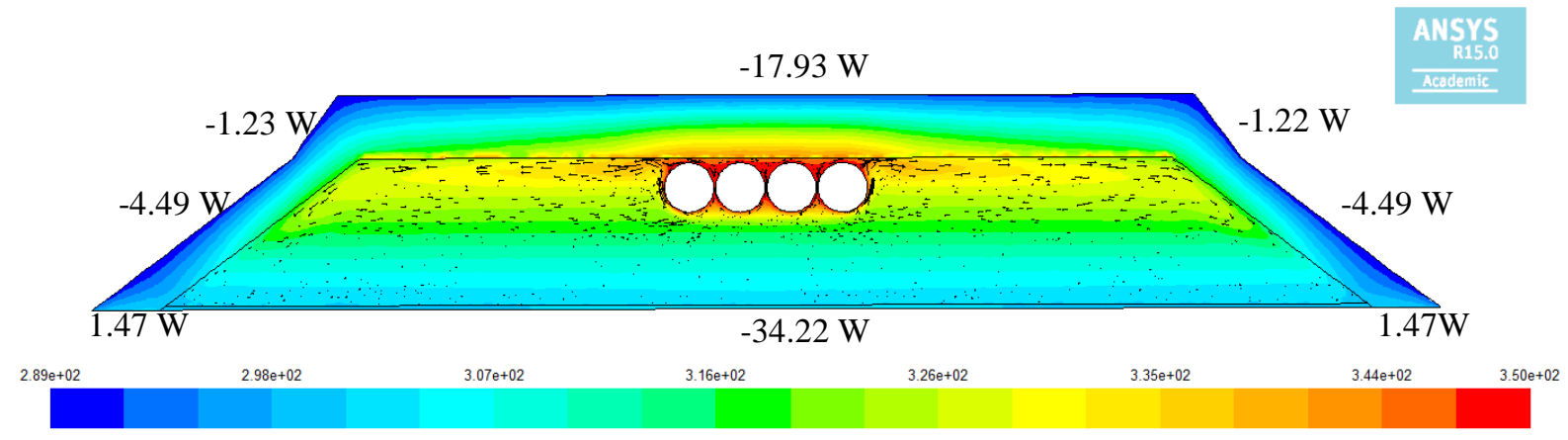

(b) Maximum velocity 7.91E $-2 \mathrm{~m} / \mathrm{s}$ and minimum velocity $3.12 \mathrm{E}-5 \mathrm{~m} / \mathrm{s}$.

Figure 14: Temperature contours, overlaid with velocity vectors and heat losses from every each boundary in the optimum case at specified pipe temperature (a) $500 \mathrm{~K}$ (b) $350 \mathrm{~K}$.

\section{Conclusions and Future work}

\subsection{Conclusions}

Mathematical optimization is shown to be a powerful tool that engineers can use to reach engineering goals. In this paper, an LFR cavity receiver was optimized in order to find the most appropriate geometry for minimizing heat losses and side wind load. The former resulted in a more thermally efficient receiver while the latter implied that the receiver was as small as possible, reducing its cost. The multiple objectives were incorporated using a Multi-Objective Genetic Algorithm to find multiple Pareto optima. If all competing objectives were not included in a design formulation, then one objective might be optimized at the expense of other performance parameters.

Some specific conclusions can be made regarding the work: 
1. The careful selection of geometrical parameters that govern the geometry of an LFR cavity receiver allows the design optimization process the freedom to suggest candidate optimum designs that minimizes all the objectives.

2. Accurate modeling of all the heat transfer mechanisms is required in order to assess their relative importance in the optimization process. This includes accounting for natural convection. The traditional use of the Boussinesq approximation has been replaced with the more accurate incompressible ideal gas assumption to relate the density of the air in the cavity to the temperature. The insulation material is included in the model since its shape influences the local conduction, especially in the cavity corner area. The selective absorption of the glass cover is incorporated with a dual-band gray approach in the Discrete Ordinates Method. This approach allowed for the capture of the greenhouse effect caused by the glass cover.

3. The assumption of replacing the effect of solar irradiation with a specified outside temperature (and thereby assuming a uniform flux) is shown to be good one by validating the approach against a more expensive and elaborate method that incorporates a non-uniform solar heat flux distribution on the pipes.

4. The radiation properties of the cavity and absorber pipe surfaces are treated with a dual gray-band approach based on their surface roughness. This implies that they reflect radiation specularly at high wavelengths but diffusely for the rest of the spectrum. The dual band also enabled the modeling of selective coatings through the band-selective specification of surface absorptance and emissivity.

5. A limited investigation of varying the specified pipe temperature is performed to mimic the fact that an LFR cavity receiver would be operating at different temperatures depending on the location along the length of the plant. For the temperatures considered, radiation was found to be the major contributor to heat losses.

\subsection{Future work}

Other objectives will be included in the optimization formulation. An example is optical efficiency, which would influence the thermal efficiency determined in this paper. Other factors that can be considered are economic ones that relate to material cost and manufacturing considerations. Representing a new arrangement for a pipe bundle might also lead to a more efficient cavity. Partial submergence of the pipes into the top insulation or using a staggered bundle arrangement is an example. In addition, number of pipes can be defined as a new independent parameter which would give the optimization process more freedom to come up with an optimum design.

\section{Acknowledgements}

The authors wish to acknowledge funding from the University of Pretoria, South Africa, the South African National Research Foundation, as well as the Solar Spoke of the South African Department of Trade and Industry.

\section{References}

Abbas, R., Muñoz-Antón, J., Valdés, M., Martínez-Val, J.M., 2013. High concentration linear Fresnel reflectors. Energy Convers. Manage. 72, 60-68.

ANSYS, 2013a. Ansys Fluent Help, version 15, ANSYS Incorporated.

ANSYS, 2013b. Design Exploration User Guide, version 15, ANSYS Incorporated.ASME B36.19M-2004, 2004. Stainless Steel Pipe, American Society of Mechanical Engineers.

Bakker, A., 2013. Lecture 7 - Meshing, Applied Computational Fluid Dynamics, http://www.bakker.org/dartmouth06/engs150/07-mesh.pdf, Last accessed: 9.10.2013. 
Bansal, N.P., Doremus, R.H., 1986. Handbook of Glass Properties, 1st edition, Elsevier.

Bejan, A., 1993. Heat Transfer, 1st edition, John Wiley and Sons.

Bennett, H.E., Porteus, J.O., 1961. Relation Between Surface Roughness and Specular Reflectance at Normal Incidence, U.S. Naval Ordnance Test Station.

Bernhard, R., Laabs, H.-G., de Lalaing, J., Eck, M., Eickhoff, M., Pottler, K., Morin, G., Heimsath, A., Georg, A. and Häberle, A., 2008. Linear Fresnel Collector demonstration on the PSA Part 1 - Design, construction and quality control, 14th International Symposium on Concentrated Solar Power and Chemical Energy

Bhaduri, S., Murphy, L.M., 1985. Wind Loading on Solar Collectors, Solar Energy Research Institute, SERI/TR-253-2169.

Cengel, Y.A., Ghajar, A. J., 2010. Heat and Mass Transfer Fundamentals and Applications, 4th edition, McGraw-Hill.

Dhall, A., Datta, A.K., Torrance, K.E., Almeida, M.F., 2009. Radiative heat exchange modeling inside an oven, AIChE Journal: Bioengineering, Food, and Natural Products, 55, 2448-2460.

Facão, J., Oliveira, A.C., 2011. Numerical simulation of a trapezoidal cavity receiver for a linear Fresnel solar collector concentrator, Renewable Energy, 36, 90-96.

Flores Larsen, S., Altamirano, M., Hernández, A., 2012. Heat loss of a trapezoidal cavity absorber for a linear Fresnel reflecting solar concentrator, Renewable Energy, 39, 186-206.

Guo, P., Li, J., Wang, Y., 2014. Numerical simulations of solar chimney power plant with radiation model, Renewable Energy, 62, 24-30.

Haberle, A., Zahler, C., Lerchenmuller, H., Mertins, M., Wittwer, C., Trieb, F., Dersch, J., 2002. The solarmundo line focussing Fresnel collector optical and thermal performance and cost calculations, 11th SolarPACES Conference, Zurich, Switzerland.

Heimsath, A., Cuevas, F., Hofer, A. Nitz, P., Platzer, W.J., 2014. Linear fresnel collector receiver: heat loss and temperatures, Energy Procedia, 49, 386-397.

Incropera, F.P., DeWitt, D.P., Bergman, T.L., Lavine, A.S., 2006. Fundamentals of Heat and Mass Transfer, 6th Edition, John Wiley and Sons.

Lai, Y., Wu, T., Che, Sh., Dong, Z., Lyu, M., 2013. Thermal performance prediction of a trapezoidal cavity absorber for a linear Fresnel reflector, Advances in Mechanical Engineering, http://dx.doi.org/10.1155/2013/615742.

Lienhard IV, J.H., Lienhard V, J.H., 2003. A Heat Transfer Textbook, 3rd edition, Phlogiston Press.

Lin, M., Sumathy, K., Dai, Y.J., Wang, R.Z., Chen, Y., 2013. Experimental and theoretical analysis on a linear Fresnel reflector solar collector prototype with V-shaped cavity receiver. Appl. Therm. Eng. 51, 963-972.

Loenen E., Van der Tempel, L., 1996. Determination of absorption coefficients of glasses at high temperatures, by measuring the thermal emission. Unclassified report, NL-UR 020/96, Philips Electronics N.V.

Lovegrove, K., Stein, W., 2012. Concentrating Solar Power Technology: Principles, Developments and Applications, $1^{\text {st }}$ edition, Elsevier.

LS-OPT, 2003, User's Manual. s.l., version 2, Livemore Software Technology Corporation. 
Mellott, N.P., Brantley, S.L., Hamilton, J.P., Pantano, C.G., 2001. Evaluation of surface preparation methods for glass, Surf. Interface Anal. 31, 362-368.

Mills, D., 2004, Advances in solar thermal electricity technology, Sol. Energy, 76, 2004, 19-31.

Modest, M.F., 2013. Radiative Heat Transfer, 3rd edition, Elsevier.

Moghimi, M.A., Craig, K.J., Meyer J.P., 2015. A novel computational approach to combine the optical and thermal modelling of a Linear Fresnel Reflector receiver, $3^{\text {rd }}$ Southern African Solar Energy Conference, SASEC2015, 11-13 May 2015, Kruger Park, South Africa.

NREL, 2013a. Concentrating solar power projects by status, http://www.nrel.gov/csp/solarpaces/by_status.cfm, Last accessed: 24.11.2013.

NREL, 2013b. Linear Fresnel reflector projects, http://www.nrel.gov/csp/solarpaces/linear_fresnel.cfm , Last accessed: 24.11.2013.

NREL, 2014. SolTrace Optical Modeling Software, http:/www.nrel.gov/csp/soltrace , Last accessed: 2.12.2014.

Pye, J.D., Morrison, G.L., Behnia, M., 2003a. Transient modelling of cavity receiver heat transfer for the compact linear Fresnel reflector, 41st Annual Conference of ANZSES, Australia.

Pye, J.D., Morrison, G.L., Behnia, M., 2003b. Convection inside the cavity receiver of the CLFR concentrating solar power system, 7th Natural Convection Workshop, Australia.

Pye, J.D., Morrison, G.L., Behnia, M., Mills, D., 2003c. Modelling of cavity receiver heat transfer for the compact linear Fresnel reflector, ISES Solar World Congress, Sweden.

Pye, J.D., 2008, System Modelling of the Compact Linear Fresnel Reflector, PhD Thesis, University of New South Wales, Australia.

Sahoo, S.S., Singh, S., Banerjee, R., 2012. Analysis of heat losses from a trapezoidal cavity used for linear Fresnel reflector system, Sol. Energy, 86, 1313-1322.

Sahoo, S.S., Varghese, S.M., Kumar, C.S., Viswanathan, S.P., Singh, S., Banerjee, R., 2013a. Experimental investigation and computational validation of heat losses from the cavity receiver used in linear Fresnel reflector solar thermal system, Renewable Energy, 55, 18-23.

Sahoo, S.S., Singh, S., Banerjee, R., 2013b. Steady state hydrothermal analysis of the absorber tubes used in linear Fresnel reflector solar thermal system, Sol. Energy, 87, 84-95.

Singh, P.L., Sarviya, R.M., Bhagoria, J.L., 2010. Heat loss study of trapezoidal cavity absorbers for linear solar concentrating collector, Energy Convers. Manage. 51, 329-337.

TIASA, 2001, Thermal Insulation Handbook, Thermal Insulation Association of Southern Africa. 